A priori error analysis of stochastic Galerkin mixed approximations of elliptic PDEs with random data

Bespalov, Alexei and Powell, Catherine E. and Silvester, David

2012

MIMS EPrint: 2011.91

Manchester Institute for Mathematical Sciences

School of Mathematics

The University of Manchester

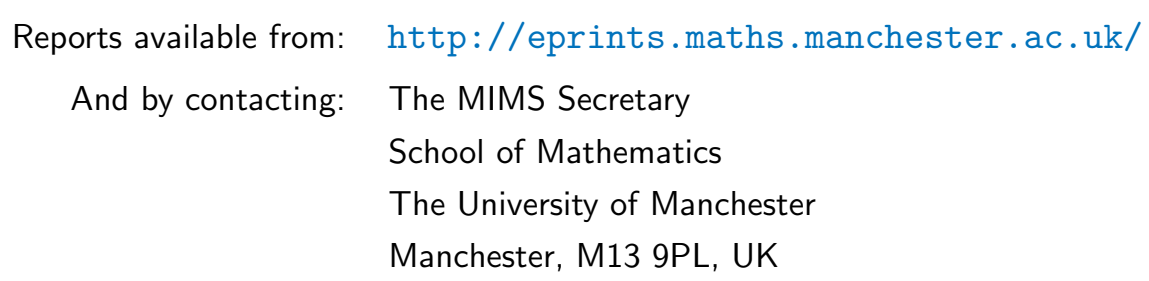

ISSN 1749-9097 


\title{
A PRIORI ERROR ANALYSIS OF STOCHASTIC GALERKIN MIXED APPROXIMATIONS OF ELLIPTIC PDEs WITH RANDOM DATA*
}

\author{
ALEXEI BESPALOV ${ }^{\dagger}$, CATHERINE E. POWELL $^{\dagger}$, AND DAVID SILVESTER ${ }^{\dagger}$
}

\begin{abstract}
We construct stochastic Galerkin approximations to the solution of a first-order system of PDEs with random coefficients. Under the standard finite-dimensional noise assumption, we transform the variational saddle point problem to a parametric deterministic one. Approximations are constructed by combining mixed finite elements on the computational domain with $M$-variate tensor product polynomials. We study the inf-sup stability and well-posedness of the continuous and finite-dimensional problems, the regularity of solutions with respect to the $M$ parameters describing the random coefficients, and establish a priori error estimates for stochastic Galerkin finite element approximations.
\end{abstract}

Key words. mixed finite elements, saddle point problems, stochastic finite elements, random data, Karhunen-Loève expansion, a priori analysis, error estimates

AMS subject classifications. 35R60, 65C20, 65N30, 65N15

DOI. $10.1137 / 110854898$

1. Introduction. The numerical approximation of solutions to elliptic PDEs with random data is a topical research area (see, e.g., [2], [3], [11], [12], [14], [19], [25]). In particular, stochastic Galerkin approximations have been extensively studied, and a priori error estimates are provided in [3]. Relatively little work has been done on saddle point problems with random data (although see [10], [17], and [20]) and a priori error analysis for such problems is much less well developed.

To begin, consider the abstract problem: find $\mathbf{q} \in \mathbf{V}$ and $u \in W$ such that

$$
\begin{aligned}
a(\mathbf{q}, \mathbf{r})+b(\mathbf{r}, u) & =\ell(\mathbf{r}) & & \forall \mathbf{r} \in \mathbf{V}, \\
b(\mathbf{q}, v) & =t(v) & & \forall v \in W,
\end{aligned}
$$

where $a(\cdot, \cdot): \mathbf{V} \times \mathbf{V} \rightarrow \mathbb{R}$ and $b(\cdot, \cdot): \mathbf{V} \times W \rightarrow \mathbb{R}$ are bounded bilinear forms, $\ell: \mathbf{V} \rightarrow \mathbb{R}, t: W \rightarrow \mathbb{R}$ are bounded linear functionals, and $\mathbf{V}$ and $W$ are Hilbert spaces of vector-valued functions and scalar functions, respectively. The general theory of saddle point problems (see [9]) guarantees that a unique solution exists if $a(\cdot, \cdot)$ is coercive on the null-space of $b(\cdot, \cdot)$, and if

$$
\exists \beta>0 \quad \text { such that } \quad \sup _{\mathbf{r} \in \mathbf{V}} \frac{b(\mathbf{r}, v)}{\|\mathbf{r}\|_{\mathbf{V}}} \geq \beta\|v\|_{W} \quad \forall v \in W .
$$

Now, let $D \subset \mathbb{R}^{d}(d=2,3)$ be a Lipschitz domain with polygonal $(d=2)$ or polyhedral $(d=3)$ boundary $\partial D$ and let $\partial D_{\text {Dir }}$ and $\partial D_{\text {Neu }}$ be disjoint open subsets of $\partial D$ such that $\partial D_{\text {Dir }} \neq \emptyset$ and $\partial D_{\text {Neu }}=\partial D \backslash \overline{\partial D}_{\text {Dir }}$. A commonly studied example of (1.1) arises from the mixed variational formulation of the boundary value problem

\footnotetext{
*Received by the editors November 11, 2011; accepted for publication (in revised form) May 28, 2012; published electronically August 15, 2012. This work was supported by EPSRC under grant $\mathrm{EP} / \mathrm{H} 021205 / 1$.

http://www.siam.org/journals/sinum/50-4/85489.html

${ }^{\dagger}$ School of Mathematics, University of Manchester, Oxford Road, Manchester, M13 9PL, United Kingdom (alexey.bespalov@manchester.ac.uk, catherine.powell@manchester.ac.uk, david.silvester@ manchester.ac.uk).
} 


$$
\begin{aligned}
& A^{-1} \mathbf{q}+\nabla u=0 \quad \text { in } D, \\
& \operatorname{div} \mathbf{q}=f \quad \text { in } D, \\
& u=g \text { on } \partial D_{\text {Dir }} \text {, } \\
& \mathbf{n} \cdot \mathbf{q}=0 \quad \text { on } \partial D_{\mathrm{Neu}} \text {. }
\end{aligned}
$$

Other examples arise from mixed variational formulations of, e.g., the Stokes and Navier-Stokes equations. In the case of $(1.3), \mathbf{V}:=\mathbf{H}_{0}(\operatorname{div}, D), W:=L^{2}(D)$,

$$
a(\mathbf{q}, \mathbf{r}):=\int_{D} A^{-1} \mathbf{q} \cdot \mathbf{r} d \mathbf{x}, \quad b(\mathbf{r}, u):=-\int_{D} u \operatorname{div} \mathbf{r} d \mathbf{x}, \quad t(v)=\int_{D} f v d \mathbf{x},
$$

and $\ell(\mathbf{r})=0$, where, as usual,

$\mathbf{H}_{0}(\operatorname{div}, D):=\left\{\mathbf{r} \in \mathbf{L}^{2}(D) ; \operatorname{div} \mathbf{r} \in L^{2}(D),\langle\mathbf{r}, \nabla v\rangle+\langle\operatorname{div} \mathbf{r}, v\rangle=0 \quad \forall v \in H_{0, \text { Dir }}^{1}(D)\right\}$

and $H_{0, \text { Dir }}^{1}(D)$ contains $H^{1}(D)$ functions having vanishing trace on $\partial D_{\text {Dir }}$. For $\mathbf{V}=$ $\mathbf{H}_{0}(\operatorname{div}, D)$ and $W=L^{2}(D)$, the inf-sup condition (1.2) is satisfied (see [9]) and there exists $\beta>0$, depending only on the domain $D$, such that

$$
\sup _{\mathbf{r} \in \mathbf{H}_{0}(\operatorname{div}, D)} \frac{\int_{D} v \operatorname{div} \mathbf{r} d \mathbf{x}}{\|\mathbf{r}\|_{\mathbf{H}(\operatorname{div}, D)}} \geq \beta\|v\|_{L^{2}(D)} \quad \forall v \in L^{2}(D) .
$$

Galerkin approximations to the solution $(\mathbf{q}, u)$ of the saddle point problem (1.1) can be obtained by replacing $\mathbf{V}$ and $W$ with finite-dimensional subspaces $\tilde{\mathbf{V}} \subset \mathbf{V}$ and $\tilde{W} \subset W$ that satisfy the discrete analogue of the inf-sup condition. For (1.3), we need $\tilde{\mathbf{V}} \subset \mathbf{H}_{0}(\operatorname{div}, D)$ and $\tilde{W} \subset L^{2}(D)$ for which there exists $\tilde{\beta}>0$, such that

$$
\sup _{\mathbf{r} \in \tilde{\mathbf{V}}} \frac{\int_{D} v \operatorname{div} \mathbf{r} d \mathbf{x}}{\|\mathbf{r}\|_{\mathbf{H}(\operatorname{div}, D)}} \geq \tilde{\beta}\|v\|_{L^{2}(D)} \quad \forall v \in \tilde{W} .
$$

Suitable pairs of finite element spaces, defined with respect to a partition of $D$, include the well known Raviart-Thomas (RT) and Brezzi-Douglas-Marini (BDM) spaces.

Suppose now that $A^{-1}$ in (1.3) is not known at every point in the computational domain; this is common in engineering applications. To accommodate such data uncertainty we can model $A^{-1}$ as a random field, i.e., as a random variable at every $\mathbf{x} \in D$. To that end, we introduce a complete probability space $(\Omega, \mathcal{F}, \mathbb{P})$, where $\Omega$ denotes the set of outcomes, $\mathcal{F}$ is a $\sigma$-algebra of events, and $\mathbb{P}: \mathcal{F} \rightarrow[0,1]$ is a probability measure. Now, if $A^{-1}=A^{-1}(\mathbf{x}, \omega), \mathbf{x} \in D, \omega \in \Omega$, the solution to (1.3) is a pair of random fields $(\mathbf{q}, u)=(\mathbf{q}(\mathbf{x}, \omega), u(\mathbf{x}, \omega))$ such that, $\mathbb{P}$-a.e. in $\Omega$,

$$
\begin{aligned}
A^{-1}(\mathbf{x}, \omega) \mathbf{q}(\mathbf{x}, \omega)+\nabla u(\mathbf{x}, \omega) & =0 & & \text { in } D, \\
\operatorname{div} \mathbf{q}(\mathbf{x}, \omega) & =f(\mathbf{x}) & & \text { in } D, \\
u(\mathbf{x}, \omega) & =g(\mathbf{x}) & & \text { on } \partial D_{\text {Dir }}, \\
\mathbf{n} \cdot \mathbf{q}(\mathbf{x}, \omega) & =0 & & \text { on } \partial D_{\text {Neu }} .
\end{aligned}
$$

The mixed variational formulation of (1.6) is also a saddle point problem of the form (1.1). However, the bilinear form $a(\cdot, \cdot)$ contains a random coefficient, and the solution and test functions are random fields.

Approximations of (1.6) are sought via stochastic collocation methods in [20] and [21] and via stochastic Galerkin methods in [18] and [17]. The emphasis in the latter works is on linear algebra and fast solvers, although a partial error analysis and 
discussion of inf-sup stability is provided in [17]. An interesting saddle point problem arises when solving PDEs on random domains using a fictitious domain approach, where randomness enters via the bilinear form $b(\cdot, \cdot)$ and not via $a(\cdot, \cdot)$. Stochastic Galerkin approximation of this problem is discussed in [10]. Here, we provide a full a priori error analysis for stochastic Galerkin approximations of (1.6), as has been done for second-order elliptic PDEs with random coefficients in [3].

The paper is organized as follows. In section 2 we derive a variational formulation of (1.6), introduce appropriate solution spaces, and verify well-posedness. We then approximate the random input $A^{-1}$ using a spectral expansion in $M$ random variables. We derive a perturbed saddle point problem, prove well-posedness, and convert to a deterministic problem with $M$ parameters. In section 3 we construct stochastic Galerkin approximations to the solution of the parametric deterministic problem, combining inf-sup stable deterministic finite element spaces on $D$ with $M$-variate tensor product polynomials. Our main results appear in sections 4 and 5 . In section 4 , we extend the work in [3] to show that both components of the solution to the parametric saddle point problem are analytic with respect to the $M$ parameters describing the random data. In section 5, we derive bounds for the error introduced by approximating the random data and the error in the stochastic Galerkin approximation arising from the choice of finite element and polynomial approximation spaces. We conclude our presentation in section 6 with some remarks and perspectives. The final section is a technical appendix. It contains auxiliary results needed for completeness but that disturb the flow of the discussion when included in the text.

2. Saddle point problem with random data. In order to set up the mixed variational formulation of (1.6), we first provide some notation and define suitable spaces of functions on $D \times \Omega$ (random fields) as well as bilinear forms.

For an integer $q \geq 1, L_{\mathbb{P}}^{q}(\Omega)$ denotes the set of real-valued random variables on the probability space $(\Omega, \mathcal{F}, \mathbb{P})$ with finite $q$ th moment. If $\xi \in L_{\mathbb{P}}^{1}(\Omega)$, the expectation $\mathbb{E}[\xi]$ is well defined, and if the probability density function $\rho_{\xi}: \mathbb{R} \rightarrow[0,+\infty)$ exists,

$$
\mathbb{E}[\xi]:=\int_{\Omega} \xi(\omega) d \mathbb{P}(\omega)=\int_{\mathbb{R}} y \rho_{\xi}(y) d y .
$$

In addition, the covariance of two random variables $\xi, \eta \in L_{\mathbb{P}}^{2}(\Omega)$ is defined as

$$
\operatorname{Cov}(\xi, \eta):=\mathbb{E}[(\xi-\mathbb{E}[\xi])(\eta-\mathbb{E}[\eta])] .
$$

We use boldface to denote vector-valued functions and spaces of such functions (e.g., $\left.\mathbf{H}^{s}(D)=\left(H^{s}(D)\right)^{d}\right)$. As usual, the associated norms and inner products are defined componentwise. The $L^{2}(D)$ inner product and norm of scalar functions on $D$ are denoted by $\langle\cdot, \cdot\rangle$ and $\|\cdot\|$, respectively, and we use the same notation for vectorvalued functions $\mathbf{u} \in \mathbf{L}^{2}(D)$. Standard notation is used for the differential operators $\nabla=\left(\partial / \partial x_{1}, \ldots, \partial / \partial x_{d}\right), \operatorname{div}=\nabla \cdot \operatorname{curl}=\nabla \times$, and the Laplace operator $\Delta=\operatorname{div} \nabla$.

Now, let $X(D)$ be a normed vector space of real-valued scalar functions on $D$ with norm $\|\cdot\|_{X}$. We can define the Bochner space

$$
L_{\mathbb{P}}^{2}(\Omega, X(D)):=\left\{v(\mathbf{x}, \omega): D \times \Omega \rightarrow \mathbb{R} ;\|v\|_{L_{\mathbb{P}}^{2}(\Omega, X(D))}<\infty\right\}
$$

of second-order scalar random fields, where

$$
\|v\|_{L_{\mathbb{P}}^{2}(\Omega, X(D))}:=\left(\mathbb{E}\left[\|v\|_{X}^{2}\right]\right)^{1 / 2}
$$


Spaces of vector-valued random fields are analogously defined. In particular, we will work with the spaces

$$
\mathcal{V}:=L_{\mathbb{P}}^{2}\left(\Omega, \mathbf{H}_{0}(\operatorname{div}, D)\right) \quad \text { and } \quad \mathcal{W}:=L_{\mathbb{P}}^{2}\left(\Omega, L^{2}(D)\right)
$$

with the corresponding norms

$$
\|\mathbf{r}\|_{\mathcal{V}}:=\left(\mathbb{E}\left[\|\mathbf{r}\|_{\mathbf{H}(\operatorname{div}, D)}^{2}\right]\right)^{1 / 2} \text { and } \quad\|v\|_{\mathcal{W}}:=\left(\mathbb{E}\left[\|v\|^{2}\right]\right)^{1 / 2},
$$

where $\|\mathbf{r}\|_{\mathbf{H}(\operatorname{div}, D)}^{2}:=\|\mathbf{r}\|^{2}+\|\operatorname{div} \mathbf{r}\|^{2}$.

We make the following assumptions on the input random field $A^{-1}$ in (1.6).

Assumption 2.1. $A^{-1}(\mathbf{x}, \omega): D \times \Omega \rightarrow \mathbb{R}$ is a second-order random field, i.e., $A^{-1}(\mathbf{x}, \cdot) \in L_{\mathbb{P}}^{2}(\Omega) \forall \mathbf{x} \in D$, with given mean $\mathbb{E}\left[A^{-1}\right](\mathbf{x})=\int_{\Omega} A^{-1}(\mathbf{x}, \omega) d \mathbb{P}(\omega) \in L^{2}(D)$ and covariance function

$$
C\left[A^{-1}\right]\left(\mathbf{x}, \mathbf{x}^{\prime}\right):=\operatorname{Cov}\left(A^{-1}(\mathbf{x}, \cdot), A^{-1}\left(\mathbf{x}^{\prime}, \cdot\right)\right) \in L^{2}(D \times D) .
$$

Assumption 2.2. $A^{-1}(\mathbf{x}, \omega) \in L^{\infty}(D \times \Omega)$ is uniformly bounded away from zero, i.e., there exist positive constants $C_{\min }$ and $C_{\max }$ such that

$$
0<C_{\min } \leq A^{-1}(\mathbf{x}, \omega) \leq C_{\max }<\infty \quad \text { a.e. in } D \times \Omega .
$$

We now define two bilinear forms,

$$
\begin{aligned}
a(\mathbf{q}, \mathbf{r}) & :=\mathbb{E}\left[\int_{D} A^{-1}(\mathbf{x}, \cdot) \mathbf{q}(\mathbf{x}, \cdot) \cdot \mathbf{r}(\mathbf{x}, \cdot) d \mathbf{x}\right], \quad \mathbf{q}, \mathbf{r} \in \mathcal{V} \\
b(\mathbf{r}, v) & :=-\mathbb{E}\left[\int_{D} v(\mathbf{x}, \cdot) \operatorname{div} \mathbf{r}(\mathbf{x}, \cdot) d \mathbf{x}\right], \quad \mathbf{r} \in \mathcal{V}, v \in \mathcal{W},
\end{aligned}
$$

and, for simplicity, assume $g \equiv 0$ on $\partial D_{\text {Dir }}$ in (1.6). Then, for a given $f \in L^{2}(D)$, the variational formulation of problem (1.6) reads as follows: find $\mathbf{q} \in \mathcal{V}$ and $u \in \mathcal{W}$ such that

$$
\begin{array}{rlrl}
a(\mathbf{q}, \mathbf{r})+b(\mathbf{r}, u) & =0 & & \forall \mathbf{r} \in \mathcal{V} \\
b(\mathbf{q}, v) & =-\mathbb{E}[\langle f, v\rangle] & \forall v \in \mathcal{W} .
\end{array}
$$

The well-posedness of (2.6) is established in the next lemma.

Lemma 2.1. If $A^{-1}(\mathbf{x}, \omega)$ satisfies Assumption 2.2, then problem (2.6) admits a unique solution pair $(\mathbf{q}, u) \in \mathcal{V} \times \mathcal{W}$. Moreover,

$$
\begin{gathered}
\|\mathbf{q}\|_{L_{\mathbb{P}}^{2}\left(\Omega, \mathbf{L}^{2}(D)\right)} \leq \frac{C_{\max }}{\beta C_{\min }}\|f\|, \\
\|\mathbf{q}\| \mathcal{V} \leq\left(\left(\frac{C_{\max }}{C_{\min } \beta}\right)^{2}+1\right)^{1 / 2}\|f\|, \quad\|u\|_{\mathcal{W}} \leq \frac{C_{\max }^{2}}{C_{\min } \beta^{2}}\|f\|,
\end{gathered}
$$

where $C_{\min }, C_{\max }$ are as in (2.3) and $\beta>0$ satisfies (1.4) and is also the inf-sup constant for (2.6).

The proof follows the general theory of saddle point problems in [9, Chapter II]. We skip it here as is it essentially the same as the proof of Lemma 2.3, given later. 
Remark 2.1. It is possible to guarantee the existence and uniqueness of the solution to problem (2.6) under a weaker assumption on $A^{-1}$. More precisely, one can assume, instead of (2.3), that $\mathbb{P}$-a.e. in $\Omega$ there holds

$$
0<C_{\min }(\omega) \leq A^{-1}(\mathbf{x}, \omega) \leq C_{\max }(\omega)<\infty \quad \text { a.e. in } D
$$

(so $C_{\min }$ and $C_{\max }$ are random variables). An assumption of this type is advantageous when $A^{-1}$ is an unbounded (e.g., Gaussian or lognormal) random variable at $\mathbf{x} \in D$. (We do not consider that case here.) See [2, Lemma 1.2] and [11, 12] for a discussion of a similar assumption for primal formulations of elliptic PDEs with random data.

2.1. A perturbed saddle point problem with random data. In order to transform the saddle point problem (2.6) into one that is amenable to solution by deterministic numerical methods, we use a spectral expansion of $A^{-1}(\mathbf{x}, \omega)$ to separate out the dependence on $\mathbf{x} \in D$ and $\omega \in \Omega$. Several such representations are available (see [25] for a survey). Herein, we focus on the Karhunen-Loève (KL) expansion

$$
A^{-1}(\mathbf{x}, \omega)=\mathbb{E}\left[A^{-1}\right](\mathbf{x})+\sum_{j=1}^{\infty} \sqrt{\lambda_{j}} \varphi_{j}(\mathbf{x}) \xi_{j}(\omega)
$$

(see [26]), where $\left\{\xi_{j}\right\}_{j=1}^{\infty}$ is a family of uncorrelated random variables with mean zero and unit variance, and $\left\{\left(\lambda_{j}, \varphi_{j}\right)\right\}_{j=1}^{\infty}$, with $\lambda_{1} \geq \lambda_{2} \geq \ldots \geq 0$, are the ordered eigenpairs of the integral operator $\mathcal{C}_{A^{-1}}: L^{2}(D) \rightarrow L^{2}(D)$, defined by

$$
\left(\mathcal{C}_{A^{-1}} w\right)(\mathbf{x})=\int_{D} C\left[A^{-1}\right]\left(\mathbf{x}, \mathbf{x}^{\prime}\right) w\left(\mathbf{x}^{\prime}\right) d \mathbf{x}^{\prime} .
$$

The first step in discretizing (2.6) is to approximate $A^{-1}$ by truncating (2.9) after $M$ terms:

$$
A^{-1}(\mathbf{x}, \omega) \approx A_{M}^{-1}(\mathbf{x}, \omega):=\mathbb{E}\left[A^{-1}\right](\mathbf{x})+\sum_{j=1}^{M} \sqrt{\lambda_{j}} \varphi_{j}(\mathbf{x}) \xi_{j}(\omega), \quad M \in \mathbb{N} .
$$

Using the truncated coefficient $A_{M}^{-1}(\mathbf{x}, \omega)$ instead of $A^{-1}(\mathbf{x}, \omega)$ in (2.6), we arrive at a perturbed problem: find $\mathbf{q}^{(M)} \in \mathcal{V}$ and $u^{(M)} \in \mathcal{W}$ such that

$$
\begin{aligned}
a_{M}\left(\mathbf{q}^{(M)}, \mathbf{r}\right)+b\left(\mathbf{r}, u^{(M)}\right) & =0 & & \forall \mathbf{r} \in \mathcal{V}, \\
b\left(\mathbf{q}^{(M)}, v\right) & =-\mathbb{E}[\langle f, v\rangle] & & \forall v \in \mathcal{W},
\end{aligned}
$$

where now the leading bilinear form in the first equation is defined by

$$
a_{M}(\mathbf{q}, \mathbf{r}):=\mathbb{E}\left[\int_{D} A_{M}^{-1}(\mathbf{x}, \cdot) \mathbf{q}(\mathbf{x}, \cdot) \cdot \mathbf{r}(\mathbf{x}, \cdot) d \mathbf{x}\right], \quad \mathbf{q}, \mathbf{r} \in \mathcal{V}
$$

It follows immediately from Lemma 2.1 that (2.12) is uniquely solvable if $A_{M}^{-1}$ is uniformly bounded almost everywhere in $D \times \Omega$, i.e., if there exist positive constants $\underline{C}_{M}$ and $\bar{C}_{M}$ (depending on $M$ ) such that $\underline{C}_{M} \leq A_{M}^{-1}(\mathrm{x}, \omega) \leq \bar{C}_{M}$ a.e. in $D \times \Omega$. However, it is possible to bypass this requirement if $\left\|A^{-1}-A_{M}^{-1}\right\|_{L^{\infty}(D \times \Omega)} \rightarrow 0$ as $M \rightarrow \infty$. To ensure this, we need the following assumption.

Assumption 2.3. The family $\boldsymbol{\xi}=\left\{\xi_{j}\right\}_{j=1}^{\infty}$ of random variables in the KL expansion (2.9) is uniformly bounded, i.e., $\exists C_{\boldsymbol{\xi}}>0$ such that $\left\|\xi_{j}\right\|_{L_{\mathbb{P}}^{\infty}(\Omega)} \leq C_{\boldsymbol{\xi}} \forall j \geq 1$. 
Now, for KL expansions with bounded random variables, the rate at which the error $\left\|A^{-1}-A_{M}^{-1}\right\|_{L^{\infty}(D \times \Omega)}$ converges to zero depends on the decay rate of the eigenvalues $\lambda_{j}$. Studies of decay rates of eigenvalues of integral operators such as the one in (2.10) and the dependence on the regularity of the kernel (here, the covariance function) date back to [31] (see also [24], [28], and [19]). In general, the smoother the covariance, the faster the eigenvalues decay, and the faster the truncation error $\left\|A^{-1}-A_{M}^{-1}\right\|_{L^{\infty}(D \times \Omega)}$ converges to zero. In particular, [19] shows that piecewise analytic covariance kernels lead to exponential convergence (with respect to $M$ ), whereas piecewise smooth covariance functions ensure only algebraic convergence. This result is formulated precisely in Lemma 2.2, which we quote from [19, Proposition 4.2]. First, we need the following definition.

Definition 2.1. The function $C\left[A^{-1}\right]: D \times D \rightarrow \mathbb{R}$ is piecewise analytic (resp., piecewise smooth) on $D \times D$ if there exists a finite family $\left\{D_{i}\right\}_{i=1}^{\mathcal{I}} \subset \mathbb{R}^{d}$ of mutually disjoint open subdomains of $D$ such that $\bar{D} \subseteq \cup_{i=1}^{\mathcal{I}} \bar{D}_{i}$ and for any pair $\left(i, i^{\prime}\right)$, the restriction $\left.C\left[A^{-1}\right]\right|_{D_{i} \times D_{i^{\prime}}}$ has analytic (resp., smooth) continuation in a neighborhood of $\bar{D}_{i} \times \bar{D}_{i^{\prime}}$.

Lemma 2.2. Let Assumption 2.3 hold. If $C\left[A^{-1}\right]$ is piecewise analytic on $D \times D$, then there exists a constant $c>0$ such that for any $\ell>0$ there holds

$$
\left\|A^{-1}-A_{M}^{-1}\right\|_{L^{\infty}(D \times \Omega)} \leq C e^{-c(1 / 2-\ell) M^{1 / d}} \quad \forall M \in \mathbb{N}
$$

with a positive constant $C$ depending on $d, \ell, c, C \boldsymbol{\xi}$, and $\mathcal{I}$.

If $C\left[A^{-1}\right]$ is piecewise smooth on $D \times D$, then for any $t, \ell>0$ there holds

$$
\left\|A^{-1}-A_{M}^{-1}\right\|_{L^{\infty}(D \times \Omega)} \leq C M^{1-t(1-\ell) / d} \quad \forall M \in \mathbb{N}
$$

with $C>0$ depending on $d, t, \ell, C \xi$, and $\mathcal{I}$.

Corollary 2.1. Let $A^{-1}(\mathbf{x}, \omega)$ satisfy Assumptions 2.1, 2.2, and 2.3. If the covariance function $C\left[A^{-1}\right]$ is piecewise smooth on $D \times D$, then there exists $M_{0} \in \mathbb{N}$ depending on $C_{\min }, C_{\max }$ in (2.3) and on $C\left[A^{-1}\right]$ such that for any $M \geq M_{0}$,

$$
\alpha_{\min }:=\frac{1}{2} C_{\min } \leq A_{M}^{-1}(\mathbf{x}, \omega) \leq C_{\max }+\frac{1}{2} C_{\min }=: \alpha_{\max } \quad \text { a.e. in } D \times \Omega .
$$

Hence, problem (2.12) admits a unique solution $\left(\mathbf{q}^{(M)}, u^{(M)}\right) \in \mathcal{V} \times \mathcal{W}$ satisfying

$$
\left\|\mathbf{q}^{(M)}\right\|_{\mathcal{V}} \leq\left(\left(\frac{\alpha_{\max }}{\alpha_{\min } \beta}\right)^{2}+1\right)^{1 / 2}\|f\|, \quad\left\|u^{(M)}\right\|_{\mathcal{W}} \leq \frac{\alpha_{\max }^{2}}{\alpha_{\min } \beta^{2}}\|f\|,
$$

where $\beta>0$ satisfies (1.4) and is also the inf-sup constant for (2.12).

2.2. Parametric deterministic reformulation. We are now going to write problem (2.12) in an equivalent parametric form. This is straightforward under the following assumption on the random variables $\xi_{j}$ in the KL expansion (2.9).

Assumption 2.4. The random variables $\xi_{j}: \Omega \rightarrow \mathbb{R}(j=1,2, \ldots)$ in $(2.9)$ are independent, $\Gamma_{j}:=\xi_{j}(\Omega)$ is a bounded interval in $\mathbb{R}$, and the density function $\rho_{j}$ : $\Gamma_{j} \rightarrow \mathbb{R}^{+}$of each $\xi_{j}$ is given.

When using $A_{M}^{-1}(\mathbf{x}, \omega)$ in $(2.12)$, we restrict the variability of $A^{-1}$ to the $M$ random variables $\xi_{1}, \ldots, \xi_{M}$. Since $\mathbf{q}^{(M)}$ and $u^{(M)}$ exist, and are measurable with respect to the $\sigma$-algebra generated by $\left(\xi_{1}, \ldots, \xi_{M}\right)$, the Doob-Dynkin lemma (cf. [27]) says that $\mathbf{q}^{(M)}$ and $u^{(M)}$ are functions of these same $M$ random variables. With a slight abuse of notation, we can write $\mathbf{q}^{(M)}(\mathbf{x}, \omega)=\mathbf{q}^{(M)}\left(\mathbf{x}, \xi_{1}(\omega), \ldots, \xi_{M}(\omega)\right)$ and 
$u^{(M)}(\mathbf{x}, \omega)=u^{(M)}\left(\mathbf{x}, \xi_{1}(\omega), \ldots, \xi_{M}(\omega)\right)$. Now, let $\mathbf{y}=\left(y_{1}, \ldots, y_{M}\right) \in \Gamma$ be the vector whose components are $y_{j}=\xi_{j}(\omega), j=1, \ldots, M$, with $\Gamma:=\Gamma_{1} \times \cdots \times \Gamma_{M} \subset \mathbb{R}^{M}$. By independence of the $\xi_{j}$, the joint probability density of $\left(\xi_{1}, \ldots, \xi_{M}\right)$ is $\rho(\mathbf{y}):=$ $\prod_{j=1}^{M} \rho_{j}\left(y_{j}\right)$, and for any measurable function $g$ of $\xi_{1}, \ldots, \xi_{M}, \mathbb{E}[g]=\int_{\Gamma} \rho(\mathbf{y}) g(\mathbf{y}) d \mathbf{y}$.

We can now write the parametric variational formulation equivalent to (2.12) as follows: find $\left(\mathbf{q}^{(M)}, u^{(M)}\right) \in \mathbf{V} \times W$ satisfying

$$
\begin{aligned}
a_{M}\left(\mathbf{q}^{(M)}, \mathbf{r}\right)+b\left(\mathbf{r}, u^{(M)}\right) & =0 & & \forall \mathbf{r} \in \mathbf{V}, \\
b\left(\mathbf{q}^{(M)}, v\right) & =-\int_{\Gamma}\langle f, v\rangle \rho(\mathbf{y}) d \mathbf{y} & & \forall v \in W,
\end{aligned}
$$

where $\mathbf{V}:=L_{\rho}^{2}\left(\Gamma ; \mathbf{H}_{0}(\operatorname{div}, D)\right), W:=L_{\rho}^{2}\left(\Gamma ; L^{2}(D)\right)$ are the Bochner spaces with norms

$$
\|\mathbf{r}\|_{\mathbf{V}}^{2}:=\left(\int_{\Gamma}\|\mathbf{r}(\cdot, \mathbf{y})\|_{\mathbf{H}(\operatorname{div}, D)}^{2} \rho(\mathbf{y}) d \mathbf{y}\right), \quad\|v\|_{W}^{2}:=\left(\int_{\Gamma}\|v(\cdot, \mathbf{y})\|_{L^{2}(D)}^{2} \rho(\mathbf{y}) d \mathbf{y}\right) .
$$

The bilinear forms in (2.18) are now rewritten as

$$
\begin{aligned}
a_{M}(\mathbf{q}, \mathbf{r}) & =\int_{\Gamma} \rho(\mathbf{y}) \int_{D} A_{M}^{-1}(\mathbf{x}, \mathbf{y}) \mathbf{q}(\mathbf{x}, \mathbf{y}) \cdot \mathbf{r}(\mathbf{x}, \mathbf{y}) d \mathbf{x} d \mathbf{y}, \quad \mathbf{q}, \mathbf{r} \in \mathbf{V}, \\
b(\mathbf{r}, v) & =-\int_{\Gamma} \rho(\mathbf{y}) \int_{D} v(\mathbf{x}, \mathbf{y}) \operatorname{div} \mathbf{r}(\mathbf{x}, \mathbf{y}) d \mathbf{x} d \mathbf{y}, \quad \mathbf{r} \in \mathbf{V}, v \in W,
\end{aligned}
$$

where $a_{M}(\cdot, \cdot)$ contains the parameterized coefficient

$$
A_{M}^{-1}(\mathbf{x}, \mathbf{y})=\mathbb{E}\left[A^{-1}\right](\mathbf{x})+\sum_{j=1}^{M} \sqrt{\lambda_{j}} \varphi_{j}(\mathbf{x}) y_{j} .
$$

In what follows, we assume $M$ is sufficiently large that $A_{M}^{-1}(\mathbf{x}, \mathbf{y})$ is bounded as in (2.16), i.e.,

$$
\alpha_{\min } \leq A_{M}^{-1}(\mathbf{x}, \mathbf{y}) \leq \alpha_{\max } \quad \text { a.e. in } D \times \Gamma,
$$

with $\alpha_{\min }=\frac{1}{2} C_{\min }$ and $\alpha_{\max }=C_{\max }+\frac{1}{2} C_{\min }$, where $C_{\min }, C_{\max }$ are as in (2.3). (See Corollary 2.1 for the necessary assumptions for (2.22) to hold.)

The following lemma establishes the well-posedness of (2.18).

Lemma 2.3. If $A^{-1}$ satisfies the assumptions in Corollary 2.1 and Assumption 2.4, then problem (2.18) admits a unique solution pair $\left(\mathbf{q}^{(M)}, u^{(M)}\right) \in \mathbf{V} \times W$. Moreover,

$$
\left\|\mathbf{q}^{(M)}\right\|_{\mathrm{V}} \leq\left(\left(\frac{\alpha_{\max }}{\alpha_{\min } \beta}\right)^{2}+1\right)^{1 / 2}\|f\|, \quad\left\|u^{(M)}\right\|_{W} \leq \frac{\alpha_{\max }^{2}}{\alpha_{\min } \beta^{2}}\|f\|,
$$

where $\beta>0$ satisfies (1.4) and is the inf-sup constant for (2.18).

Proof. In order to apply the general result for saddle point problems (see Lemma 7.1), one needs to verify the continuity of both bilinear forms (2.19)-(2.20), the coercivity of $a_{M}(\cdot, \cdot)$ on the null-space associated with $b(\cdot, \cdot)$, and the inf-sup condition. The proof of the continuity and coercivity properties is standard (see [6, Lemma 2.3] for details). To verify the inf-sup condition, for any $v \in W$ we use Lemma 7.2 to find $\mathbf{z} \in \mathbf{V}$ such that $\operatorname{div} \mathbf{z}=v$ in $W$ and $\|\mathbf{z}\|_{\mathbf{V}} \leq C_{D}\|v\|_{W}$, where $C_{D}$ is a constant depending only on $D$. Then, the inf-sup condition follows:

$$
\sup _{\mathbf{r} \in \mathbf{V}} \frac{b(\mathbf{r}, v)}{\|\mathbf{r}\|_{\mathbf{V}}} \geq \frac{-b(\mathbf{z}, v)}{\|\mathbf{z}\|_{\mathbf{V}}}=\frac{\int_{\Gamma}\langle v, \operatorname{div} \mathbf{z}\rangle \rho(\mathbf{y}) d \mathbf{y}}{\|\mathbf{z}\|_{\mathbf{V}}}=\frac{\|v\|_{W}^{2}}{\|\mathbf{z}\|_{\mathbf{V}}} \geq \beta\|v\|_{W} \quad \forall v \in W
$$


with the constant $\beta:=1 / C_{D}$. Following the arguments in Lemma 7.2 for the deterministic spaces (i.e., $\mathbf{V}=\mathbf{H}_{0}(\operatorname{div}, D)$, and $\left.W=L^{2}(D)\right)$, it is possible to show the deterministic inf-sup condition (1.4) is satisfied with this same choice of $\beta$.

The above conditions ensure the existence and uniqueness of a solution to problem (2.18). Inequalities (2.23) are then established by using the standard techniques for saddle point problems (see [6, Lemma 2.3]).

3. Galerkin approximation. Our goal in this section is to construct inf-sup stable Galerkin approximations of the solution $\left(\mathbf{q}^{(M)}, u^{(M)}\right)$ to problem (2.18). To do this, we build finite-dimensional subspaces $\mathbf{V}_{h p, \mathbf{k}} \subset \mathbf{V}$ and $W_{h p, \mathbf{k}} \subset W$ by tensorizing standard finite element functions of $\mathbf{x} \in D$ and $M$-variate polynomials of $\mathbf{y} \in \Gamma$. In what follows, $h>0$ and $p \geq 1$ will always denote discretization parameters associated with the finite element approximation on $D$, whereas $\mathbf{k} \in \mathbb{N}_{0}^{M}$ will denote the discretization parameter for $M$-variate polynomial approximations on $\Gamma$.

Let $\mathcal{T}$ be a family of quasi-uniform shape-regular meshes $\Delta_{h}$ on $D$. Each mesh is a partition of $D$ into $d$-simplices (triangular or tetrahedral elements) $K_{j}$ such that $\bar{D}=\cup_{j=1}^{J} \bar{K}_{j}$, and $\bar{K}_{i} \cap \bar{K}_{j}(i \neq j)$ is either a common vertex or an entire edge/face or is empty. The parameter $h$ refers to the maximal diameter of elements in the mesh.

We start by choosing finite-dimensional subspaces of $\mathbf{H}_{0}(\operatorname{div}, D)$ and $L^{2}(D)$ that are inf-sup stable for the deterministic discrete saddle point problem (i.e., so that (1.5) holds). Denote by $\mathcal{P}_{p}(K)$ the set of polynomials in $\mathbf{x}$ of total degree $\leq p$ on a generic element $K$. We use two families of $\mathbf{H}(\operatorname{div}, D)$-conforming elements: the RT and BDM elements. (See Remark 5.1 regarding other types of mixed finite elements.) The corresponding spaces of degree $p \geq 1$ on the element $K$ are denoted as follows (see, e.g., $[9,29])$ :

$$
\mathcal{P}_{p}^{\mathrm{RT}}(K)=\left(\mathcal{P}_{p-1}(K)\right)^{d} \oplus \mathbf{x} \mathcal{P}_{p-1}(K) ; \quad \mathcal{P}_{p}^{\mathrm{BDM}}(K)=\left(\mathcal{P}_{p}(K)\right)^{d} .
$$

We use the unified notation $\mathcal{P}_{p}(K)$ to refer to either the RT or the BDM space on $K$ for $p \geq 1$. Then, we set

$$
\mathbf{X}_{h p}^{\text {div }}:=\left\{\mathbf{r} \in \mathbf{H}_{0}(\operatorname{div}, D) ;\left.\mathbf{r}\right|_{K} \in \mathcal{P}_{p}(K) \quad \forall K \in \Delta_{h}\right\} .
$$

Note that only one of the RT or BDM spaces is used in (3.1) for all simplices. Now, a compatible subspace of $L^{2}(D)$ (in the sense of (1.5); cf. [9]) is defined as follows:

$$
X_{h p}^{0}:=\left\{v \in L^{2}(D) ;\left.v\right|_{K} \in \mathcal{P}_{p-1}(K) \quad \forall K \in \Delta_{h}\right\} .
$$

Since $\mathbf{q}^{(M)}$ and $u^{(M)}$ depend on $\mathbf{y}=\left(y_{1}, y_{2}, \ldots, y_{M}\right)$, we also need suitable finitedimensional subspace(s) of $L_{\rho}^{2}(\Gamma)$. We will use the same subspace of $L_{\rho}^{2}(\Gamma)$ for both components of the solution. Given a multi-index $\mathbf{k}=\left(k_{1}, k_{2}, \ldots, k_{M}\right) \in \mathbb{N}_{0}^{M}$ of polynomial degrees $k_{j} \in \mathbb{N}_{0}$, we introduce the space of tensor product polynomials:

$$
S_{\mathbf{k}}=S_{\mathbf{k}}(\Gamma):=S_{k_{1}}\left(\Gamma_{1}\right) \otimes S_{k_{2}}\left(\Gamma_{2}\right) \otimes \ldots \otimes S_{k_{M}}\left(\Gamma_{M}\right) \subset L_{\rho}^{2}(\Gamma),
$$

where $S_{k_{j}}\left(\Gamma_{j}\right)=\operatorname{span}\left\{y_{j}^{\alpha_{j}} ; 0 \leq \alpha_{j} \leq k_{j}\right\} \subset L_{\rho_{j}}^{2}\left(\Gamma_{j}\right), j=1,2, \ldots, M$.

Now, choosing

$$
\mathbf{V}_{h p, \mathbf{k}}:=\mathbf{X}_{h p}^{\mathrm{div}} \otimes S_{\mathbf{k}} \quad \text { and } \quad W_{h p, \mathbf{k}}:=\mathbf{X}_{h p}^{0} \otimes S_{\mathbf{k}},
$$


we arrive at the stochastic Galerkin FEM (sGFEM) formulation for problem (2.18): find $\left(\mathbf{q}_{h p, \mathbf{k}}^{(M)}, u_{h p, \mathbf{k}}^{(M)}\right) \in \mathbf{V}_{h p, \mathbf{k}} \times W_{h p, \mathbf{k}}$ satisfying

$$
\begin{aligned}
a_{M}\left(\mathbf{q}_{h p, \mathbf{k}}^{(M)}, \mathbf{r}\right)+b\left(\mathbf{r}, u_{h p, \mathbf{k}}^{(M)}\right) & =0 & & \forall \mathbf{r} \in \mathbf{V}_{h p, \mathbf{k}}, \\
b\left(\mathbf{q}_{h p, \mathbf{k}}^{(M)}, v\right) & =-\int_{\Gamma}\langle f, v\rangle \rho(\mathbf{y}) d \mathbf{y} & & \forall v \in W_{h p, \mathbf{k}} .
\end{aligned}
$$

For convergence and error analysis, we now restrict ourselves to two spatial dimensions i.e., we assume that $D \subset \mathbb{R}^{2}$. (See Remark 5.1 on extensions to three dimensions.)

Lemma 3.1. Let $D \subset \mathbb{R}^{2}$. For any $h>0, p \geq 1$, and $\mathbf{k} \in \mathbb{N}_{0}^{M}$, the discrete problem (3.4) is uniquely solvable, and the sGFEM converges quasi-optimally, i.e.,

$$
\begin{aligned}
\left\|\mathbf{q}^{(M)}-\mathbf{q}_{h p, \mathbf{k}}^{(M)}\right\|_{\mathbf{V}} \leq(1+ & \left.\frac{\alpha_{\max }}{\alpha_{\min }}\right)\left(1+\frac{1}{\tilde{\beta}}\right) \inf _{\mathbf{r} \in \mathbf{V}_{h p, \mathbf{k}}}\left\|\mathbf{q}^{(M)}-\mathbf{r}\right\|_{\mathbf{V}} \\
\left\|u^{(M)}-u_{h p, \mathbf{k}}^{(M)}\right\|_{W} \leq & \left(1+\frac{1}{\tilde{\beta}}\right) \inf _{v \in W_{h p, \mathbf{k}}}\left\|u^{(M)}-v\right\|_{W} \\
& +\frac{\alpha_{\max }}{\tilde{\beta}}\left\|\mathbf{q}^{(M)}-\mathbf{q}_{h p, \mathbf{k}}^{(M)}\right\|_{\mathbf{V}}
\end{aligned}
$$

Here, $\left(\mathbf{q}^{(M)}, u^{(M)}\right) \in \mathbf{V} \times W$ is the solution of $(2.18),\left(\mathbf{q}_{h p, \mathbf{k}}^{(M)}, u_{h p, \mathbf{k}}^{(M)}\right) \in \mathbf{V}_{h p, \mathbf{k}} \times W_{h p, \mathbf{k}}$ is the solution of (3.4), $\alpha_{\min }$ and $\alpha_{\max }$ are the positive constants in (2.22), and $\tilde{\beta}>0$ is the discrete inf-sup constant for (3.4) and is independent of $h, p$, and $\mathbf{k}$.

Proof. Analogously to the proof of Lemma 2.3, we verify continuity of the bilinear forms (now on the discrete subspaces), coercivity of $a_{M}(\cdot, \cdot)$ on the discrete null-space of $b(\cdot, \cdot)$, and the discrete inf-sup condition.

Continuity of the bilinear forms is established easily:

$$
\begin{array}{ll}
\left|a_{M}(\mathbf{q}, \mathbf{r})\right| \leq \alpha_{\max }\|\mathbf{q}\|_{\mathbf{v}}\left\|_{\mathbf{r}}\right\|_{\mathbf{v}} & \forall \mathbf{q}, \mathbf{r} \in \mathbf{V}_{h p, \mathbf{k}}, \\
|b(\mathbf{r}, v)| \leq\|\mathbf{r}\|_{\mathbf{v}}\|v\|_{W} & \forall \mathbf{r} \in \mathbf{V}_{h p, \mathbf{k}}, \forall v \in W_{h p, \mathbf{k}} .
\end{array}
$$

Now, we introduce the discrete null-space associated with $b(\cdot, \cdot)$ :

$$
\mathbf{V}_{h p, \mathbf{k}}^{0}:=\left\{\mathbf{r} \in \mathbf{V}_{h p, \mathbf{k}} ; b(\mathbf{r}, v)=0 \quad \forall v \in W_{h p, \mathbf{k}}\right\} .
$$

Recalling that $b(\mathbf{r}, v)=-\int_{\Gamma}\langle v(\cdot, \mathbf{y}), \operatorname{div} \mathbf{r}(\cdot, \mathbf{y})\rangle \rho(\mathbf{y}) d \mathbf{y}$ and observing that

$$
\operatorname{div} \mathbf{r}(\mathbf{x}, \mathbf{y}) \in W_{h p, \mathbf{k}} \quad \forall \mathbf{r} \in \mathbf{V}_{h p, \mathbf{k}}
$$

we have

$$
\|\mathbf{r}\|_{\mathbf{V}}^{2}=\int_{\Gamma}\|\mathbf{r}(\cdot, \mathbf{y})\|^{2} \rho(\mathbf{y}) d \mathbf{y} \quad \forall \mathbf{r} \in \mathbf{V}_{h p, \mathbf{k}}^{0}
$$

The coercivity of $a_{M}(\cdot, \cdot)$ on $\mathbf{V}_{h p, \mathbf{k}}^{0}$ follows by making use of the lower bound in (2.22):

$$
\begin{aligned}
a_{M}(\mathbf{r}, \mathbf{r}) & =\int_{\Gamma} \rho(\mathbf{y}) \int_{D} A_{M}^{-1}(\mathbf{x}, \mathbf{y})|\mathbf{r}(\mathbf{x}, \mathbf{y})|^{2} d \mathbf{x} d \mathbf{y} \\
& \geq \alpha_{\min } \int_{\Gamma}\|\mathbf{r}(\cdot, \mathbf{y})\|^{2} \rho(\mathbf{y}) d \mathbf{y}=\alpha_{\min }\|\mathbf{r}\|_{\mathbf{V}}^{2} \quad \forall \mathbf{r} \in \mathbf{V}_{h p, \mathbf{k}}^{0}
\end{aligned}
$$


It remains to prove the discrete inf-sup condition. To that end, for any $v \in W_{h p, \mathbf{k}} \subset$ $L^{2}(D) \otimes S_{\mathbf{k}}(\Gamma)$ we use Lemma 7.2 to find $\mathbf{z} \in\left(\mathbf{H}^{\varepsilon}(D) \cap \mathbf{H}_{0}(\operatorname{div}, D)\right) \otimes S_{\mathbf{k}}(\Gamma)$ for some $\varepsilon>0$ such that $\operatorname{div} \mathbf{z}=v$ and

$$
\|\mathbf{z}\|_{L_{\rho}^{2}\left(\Gamma, \mathbf{H}^{\varepsilon}(D)\right)}^{2}=\int_{\Gamma}\|\mathbf{z}\|_{\mathbf{H}^{\varepsilon}(D)}^{2} \rho(\mathbf{y}) d \mathbf{y} \leq C_{\mathrm{reg}}^{2}\|v\|_{W}^{2} .
$$

Next, we employ the $\mathbf{H}(\operatorname{div}, D)$-conforming $h p$-interpolation operator $\Pi_{h p}^{\mathrm{div}}: \mathbf{H}^{\varepsilon}(D) \cap$ $\mathbf{H}_{0}(\operatorname{div}, D) \rightarrow \mathbf{X}_{h p}^{\text {div }}($ see section 7$)$ to define $\mathbf{z}_{h p}(\mathbf{x}, \mathbf{y}):=\Pi_{h p}^{\operatorname{div}} \mathbf{z}(\mathbf{x}, \mathbf{y}) \in \mathbf{X}_{h p}^{\text {div }} \otimes S_{\mathbf{k}}=$ $\mathbf{V}_{h p, \mathbf{k}}$. Using the properties of $\Pi_{h p}^{\text {div }}$ (see Lemma 7.3) and recalling that $\operatorname{div} \mathbf{z}=v$, we find

$$
\operatorname{div} \mathbf{z}_{h p}=\operatorname{div}\left(\Pi_{h p}^{\operatorname{div}} \mathbf{z}\right)=\Pi_{h p}^{0}(\operatorname{div} \mathbf{z})=\Pi_{h p}^{0} v=v \quad \forall v \in W_{h p, \mathbf{k}},
$$

and the following estimate holds:

$$
\begin{gathered}
\left\|\mathbf{z}_{h p}\right\|_{\mathbf{V}}^{2}=\left\|\mathbf{z}_{h p}\right\|_{L_{\rho}^{2}(\Gamma, \mathbf{H}(\operatorname{div}, D))}^{2} \leq C_{\mathrm{int}}^{2}\left(\|\mathbf{z}\|_{L_{\rho}^{2}\left(\Gamma, \mathbf{H}^{\varepsilon}(D)\right)}^{2}+\|\operatorname{div} \mathbf{z}\|_{W}^{2}\right) \\
\stackrel{(3.11)}{\leq} C_{\mathrm{int}}^{2}\left(1+C_{\mathrm{reg}}^{2}\right)\|v\|_{W}^{2},
\end{gathered}
$$

where $C_{\text {int }}>0$ is the stability constant (independent of $h$ and $p$ ) for $\Pi_{h p}^{\text {div }}$ (cf. property (1) in Lemma 7.3). Now, the inf-sup stability follows in a standard way:

$$
\sup _{\mathbf{r} \in \mathbf{V}_{h p, \mathbf{k}}} \frac{b(\mathbf{r}, v)}{\|\mathbf{r}\|_{\mathbf{V}}} \geq \frac{\int_{\Gamma}\left\langle v, \operatorname{div} \mathbf{z}_{h p}\right\rangle \rho(\mathbf{y}) d \mathbf{y}}{\left\|\mathbf{z}_{h p}\right\|_{\mathbf{V}}}=\frac{\|v\|_{W}^{2}}{\left\|\mathbf{z}_{h p}\right\|_{\mathbf{v}}} \geq \tilde{\beta}\|v\|_{W} \quad \forall v \in W_{h p, \mathbf{k}},
$$

where $\tilde{\beta}=\left(C_{\text {int }} \sqrt{1+C_{\text {reg }}^{2}}\right)^{-1}$. This finishes the proof of well-posedness.

Having identified the continuity, coercivity, and discrete inf-sup constants (see inequalities (3.7), (3.8), (3.10), and (3.13)), the estimates in (3.5)-(3.6) immediately follow from the abstract theory of saddle point problems (cf. [9, section II.2.2]).

Remark 3.1. The fact that $S_{\mathbf{k}}(\Gamma) \subset L_{\rho}^{2}(\Gamma)$ is used for both components of the solution (see (3.3)) ensures that $\operatorname{div} \mathbf{V}_{h p, \mathbf{k}}=W_{h p, \mathbf{k}}$. Consequently, the discrete inf-sup constant (see (3.13)) for (3.4) also satisfies (1.5) when we employ $\mathbf{X}_{h p}^{\text {div }} \subset \mathbf{H}_{0}(\operatorname{div}, D)$ and $X_{h p}^{0} \subset L^{2}(D)$. That is, $\tilde{\beta}$ is identical to the discrete inf-sup constant for the corresponding deterministic problem. Note also that the well-posedness proof does not depend on the choice of subspace of $L_{\rho}^{2}(\Gamma)$. Polynomials of a fixed total degree can be used instead of tensor product polynomials.

4. Regularity of the solution. Before deriving error estimates for the sGFEM solution, we study the regularity of the solution $\left(\mathbf{q}^{(M)}, u^{(M)}\right)$ to $(2.18)$ with respect to $y_{1}, \ldots, y_{M}$. In the analysis below, we follow the notation conventions from [3]. Note that the regularity of $\left(\mathbf{q}^{(M)}, u^{(M)}\right)$ with respect to $\mathbf{x} \in D$ for a fixed $\mathbf{y} \in \Gamma$ follows from the theory of deterministic elliptic boundary value problems (see, e.g., $[13,22])$. Specifically, if $\mathbb{E}\left[A^{-1}\right] \in C^{1}(\bar{D})$ and $C\left[A^{-1}\right]$ is smooth on $D \times D$, then the samples $A_{M}^{-1}(\mathbf{x}, \mathbf{y}) \in C^{1}(\bar{D})$ a.e. on $\Gamma \ni \mathbf{y}$, and hence there exists $s>0$ (depending on the geometry of $D$ and on the regularity of the right-hand side function $f$ ) such that $\left(\mathbf{q}^{(M)}(\cdot, \mathbf{y}), u^{(M)}(\cdot, \mathbf{y})\right) \in \mathbf{H}^{s}(\operatorname{div}, D) \times H^{s}(D)$ a.e. on $\Gamma$. Recall here

$$
\mathbf{H}^{s}(\operatorname{div}, D):=\left\{\mathbf{r} \in \mathbf{H}^{s}(D) ; \operatorname{div} \mathbf{r} \in H^{s}(D)\right\} .
$$

Hence, $\left(\mathbf{q}^{(M)}, u^{(M)}\right) \in L_{\rho}^{2}\left(\Gamma ; \mathbf{H}^{s}(\operatorname{div}, D)\right) \times L_{\rho}^{2}\left(\Gamma ; H^{s}(D)\right)$. 
The next lemma states that both components of the solution to problem (2.18) are analytic with respect to the $M$ parameters describing the random data. To prove this result we show that for any $j \in\{1, \ldots, M\}, \mathbf{q}^{(M)}$ and $u^{(M)}$ can be represented as power series in $y_{j}$. The corresponding coefficents in these series are coupled through a set of deterministic saddle point problems, which define the coefficients uniquely. Using upper bounds for the coefficients, we establish the convergence interval for each series (in fact, this is the same for both of them). Thus, the power series representation allows us to extend $\mathbf{q}^{(M)}$ and $u^{(M)}$ analytically (as functions of $y_{j}$ ) to a region in the complex plane.

We will assume, without loss of generality, that $\Gamma_{j}=[-1,1]$ for any $j \in\{1, \ldots, M\}$. The proof of Lemma 4.1 for other bounded intervals $\Gamma_{j} \subset \mathbb{R}$ follows easily by using an affine transformation $[-1,1] \rightarrow \Gamma_{j}$ and standard scaling.

Lemma 4.1. Let the random coefficient $A^{-1}(\mathbf{x}, \omega)$ satisfy Assumptions 2.1-2.4, and assume that the covariance function $C\left[A^{-1}\right]$ is piecewise smooth on $D \times D$. Let $\left(\mathbf{q}^{(M)}, u^{(M)}\right)$ be the solution to problem (2.18). Then, for each $j \in\{1, \ldots, M\}$, the functions $\mathbf{q}^{(M)}$ and $u^{(M)}$, as functions of the variable $y_{j}$, can be analytically extended to the following region in the complex plane:

$$
\Sigma_{j}:=\left\{z \in \mathbb{C} ;\left|z-y_{j}^{0}\right|<\frac{\inf _{\mathbf{x} \in D}\left(A_{M}^{-1}\left(\mathbf{x}, y_{j}^{0}, \mathbf{y}_{j}^{*}\right)\right)}{\sqrt{\lambda_{j}}\left\|\varphi_{j}\right\|_{L^{\infty}(D)}} \quad \forall y_{j}^{0} \in \Gamma_{j}\right\},
$$

where $\mathbf{y}_{j}^{*}=\left(y_{1}, \ldots, y_{j-1}, y_{j+1}, \ldots, y_{M}\right) \in \Gamma_{j}^{*}=\Gamma_{1} \times \ldots \times \Gamma_{j-1} \times \Gamma_{j+1} \times \ldots \times \Gamma_{M}$.

Proof. Without loss of generality we consider only $j=1$. Let us fix an arbitrary $\mathbf{y}_{1}^{*} \in \Gamma_{1}^{*}=[-1,1]^{M-1}$. For any $y_{1}^{0} \in[-1,1]$, consider two formal power series in $y_{1}$ :

$$
\mathbf{q}_{F}\left(\cdot, y_{1}\right)=\sum_{n=0}^{\infty}\left(y_{1}-y_{1}^{0}\right)^{n} \mathbf{q}_{n} \quad \text { and } \quad u_{F}\left(\cdot, y_{1}\right)=\sum_{n=0}^{\infty}\left(y_{1}-y_{1}^{0}\right)^{n} u_{n}
$$

where the coefficient pairs $\left(\mathbf{q}_{n}, u_{n}\right)$ for $n=0,1, \ldots$, are determined in a recursive way by solving the following deterministic saddle point problems:

$(n=0)$ find $\left(\mathbf{q}_{0}, u_{0}\right) \in \mathbf{H}_{0}(\operatorname{div}, D) \times L^{2}(D)$ such that

$$
\begin{gathered}
\int_{D} A_{M}^{-1}\left(\mathbf{x}, y_{1}^{0}, \mathbf{y}_{1}^{*}\right) \mathbf{q}_{0}(\mathbf{x}) \cdot \mathbf{r}(\mathbf{x}) d \mathbf{x}-\int_{D} u_{0}(\mathbf{x}) \operatorname{div} \mathbf{r}(\mathbf{x}) d \mathbf{x}=0, \\
\int_{D} v(\mathbf{x}) \operatorname{div} \mathbf{q}_{0}(\mathbf{x}) d \mathbf{x}=\int_{D} f(\mathbf{x}) v(\mathbf{x}) d \mathbf{x}
\end{gathered}
$$

for any $\mathbf{r} \in \mathbf{H}_{0}(\operatorname{div}, D)$ and $v \in L^{2}(D)$;

$(n \geq 1)$ find $\left(\mathbf{q}_{n}, u_{n}\right) \in \mathbf{H}_{0}(\operatorname{div}, D) \times L^{2}(D)$ such that

$$
\begin{aligned}
& \int_{D} A_{M}^{-1}\left(\mathbf{x}, y_{1}^{0}, \mathbf{y}_{1}^{*}\right) \mathbf{q}_{n}(\mathbf{x}) \cdot \mathbf{r}(\mathbf{x}) d \mathbf{x}-\int_{D} u_{n}(\mathbf{x}) \operatorname{div} \mathbf{r}(\mathbf{x}) d \mathbf{x}=Q_{n}^{(1)}(\mathbf{r}), \\
& \int_{D} v(\mathbf{x}) \operatorname{div} \mathbf{q}_{n}(\mathbf{x}) d \mathbf{x}=0
\end{aligned}
$$

for any $\mathbf{r} \in \mathbf{H}_{0}(\operatorname{div}, D)$ and $v \in L^{2}(D)$, where

$$
Q_{n}^{(1)}(\mathbf{r}):=-\sqrt{\lambda_{1}} \int_{D} \varphi_{1}(\mathbf{x}) \mathbf{q}_{n-1}(\mathbf{x}) \cdot \mathbf{r}(\mathbf{x}) d \mathbf{x} .
$$


Under the assumptions of the lemma, the coefficient $A_{M}^{-1}(\mathbf{x}, \mathbf{y})$ is bounded as in (2.22). Hence, the well-posedness of both (4.3) and (4.4) is established using standard arguments (see Lemma 7.1 and the proof of Lemma 2.3). Moreover, the following estimates hold for the solution $\left(\mathbf{q}_{0}, u_{0}\right)$ of problem (4.3) (cf. (2.23)):

$$
\begin{aligned}
\left\|\mathbf{q}_{0}\right\|_{\mathbf{H}(\operatorname{div}, D)} & \leq\left(\left[\frac{\sup _{\mathbf{x} \in D}\left(A_{M}^{-1}\left(\mathbf{x}, y_{1}^{0}, \mathbf{y}_{1}^{*}\right)\right)}{\beta \inf _{\mathbf{x} \in D}\left(A_{M}^{-1}\left(\mathbf{x}, y_{1}^{0}, \mathbf{y}_{1}^{*}\right)\right)}\right]^{2}+1\right)^{\frac{1}{2}}\|f\| \\
\leq & \left(\left[\frac{\alpha_{\max }}{\beta \alpha_{\min }}\right]^{2}+1\right)^{\frac{1}{2}}\|f\| \\
\left\|u_{0}\right\|_{L^{2}(D)} & \leq \frac{\alpha_{\max }}{\beta}\left\|\mathbf{q}_{0}\right\| \leq \frac{\alpha_{\max }}{\beta}\left\|\mathbf{q}_{0}\right\|_{\mathbf{H}(\operatorname{div}, D)}
\end{aligned}
$$

where $\alpha_{\min }, \alpha_{\max }$ satisfy (2.22), and $\beta$ is the inf-sup constant satisfying (1.4).

Similarly, for (4.4), we use Lemma 7.1 to estimate

$$
\begin{aligned}
\left\|\mathbf{q}_{n}\right\|_{\mathbf{H}(\operatorname{div}, D)} & \leq \frac{\left\|\sqrt{\lambda_{1}} \varphi_{1} \mathbf{q}_{n-1}\right\|_{\mathbf{H}(\operatorname{div}, D)}}{\inf _{\mathbf{x} \in D}\left(A_{M}^{-1}\left(\mathbf{x}, y_{1}^{0}, \mathbf{y}_{1}^{*}\right)\right)} \leq r_{y_{1}^{0}}^{-1}\left\|\mathbf{q}_{n-1}\right\|_{\mathbf{H}(\operatorname{div}, D)} \\
\left\|u_{n}\right\|_{L^{2}(D)} & \leq \frac{1}{\beta}\left(\frac{\sup _{\mathbf{x} \in D}\left(A_{M}^{-1}\left(\mathbf{x}, y_{1}^{0}, \mathbf{y}_{1}^{*}\right)\right)}{\inf _{\mathbf{x} \in D}\left(A_{M}^{-1}\left(\mathbf{x}, y_{1}^{0}, \mathbf{y}_{1}^{*}\right)\right)}+1\right)\left\|\sqrt{\lambda_{1}} \varphi_{1} \mathbf{q}_{n-1}\right\|_{\mathbf{H}(\operatorname{div}, D)} \\
& \leq \frac{2 \alpha_{\max }}{\beta} r_{y_{1}^{0}}^{-1}\left\|\mathbf{q}_{n-1}\right\|_{\mathbf{H}(\operatorname{div}, D)}
\end{aligned}
$$

where $r_{y_{1}^{0}}:=\frac{\inf _{\mathbf{x} \in D}\left(A_{M}^{-1}\left(\mathbf{x}, y_{1}^{0}, \mathbf{y}_{1}^{*}\right)\right)}{\sqrt{\lambda_{1}}\left\|\varphi_{1}\right\|_{L} \infty(D)}$.

Inequality (4.7) is recursive and yields

$$
\left\|\mathbf{q}_{n}\right\|_{\mathbf{H}(\operatorname{div}, D)} \leq r_{y_{1}^{0}}^{-n}\left\|\mathbf{q}_{0}\right\|_{\mathbf{H}(\operatorname{div}, D)} \quad \forall n \geq 0 .
$$

Combining (4.8) and (4.9) we find

$$
\left\|u_{n}\right\|_{L^{2}(D)} \leq \frac{2 \alpha_{\max }}{\beta} r_{y_{1}^{0}}^{-n}\left\|\mathbf{q}_{0}\right\|_{\mathbf{H}(\operatorname{div}, D)} \quad \forall n \geq 1 .
$$

Note that due to (4.6), the inequality in (4.10) is valid for $n=0$ as well. Using estimates (4.5), (4.9), and (4.10) we prove the bounds for the series in (4.2)

$$
\left\|\mathbf{q}_{F}\right\|_{\mathbf{H}(\operatorname{div}, D)} \leq C\left(\alpha_{\min }, \alpha_{\max }, \beta\right)\|f\| \sum_{n=0}^{\infty}\left|\frac{y_{1}-y_{1}^{0}}{r_{y_{1}^{0}}}\right|^{n} \leq \frac{C\|f\| r_{y_{1}^{0}}}{r_{y_{1}^{0}}-\left|y_{1}-y_{1}^{0}\right|}<+\infty
$$

and

$$
\left\|u_{F}\right\|_{L^{2}(D)} \leq \frac{\tilde{C}\left(\alpha_{\min }, \alpha_{\max }, \beta\right)\|f\| r_{y_{1}^{0}}}{r_{y_{1}^{0}}-\left|y_{1}-y_{1}^{0}\right|}<+\infty
$$

provided that $\left|y_{1}-y_{1}^{0}\right|<r_{y_{1}^{0}}$. Therefore, for any $y_{1}^{0} \in[-1,1]$, both power series in (4.2) converge in the open ball $\mathcal{B}\left(y_{1}^{0}, r_{y_{1}^{0}}\right)=\left\{y_{1} \in \mathbb{R} ;\left|y_{1}-y_{1}^{0}\right|<r_{y_{1}^{0}}\right\}$. (Convergence 
here is understood in the corresponding norm: the $\mathbf{H}(\operatorname{div}, D)$-norm for $\mathbf{q}_{F}$ and the $L^{2}(D)$-norm for $u_{F}$.) On the other hand, it is easy to check that for any $y_{1} \in \mathcal{B}\left(y_{1}^{0}, r_{y_{1}^{0}}\right)$ and for arbitrary fixed $\mathbf{y}_{1}^{*} \in[-1,1]^{M-1},\left(\mathbf{q}_{F}, u_{F}\right) \in \mathbf{H}_{0}(\operatorname{div}, D) \times L^{2}(D)$ satisfies

$$
\begin{gathered}
\int_{D} A_{M}^{-1}\left(\mathbf{x}, y_{1}, \mathbf{y}_{1}^{*}\right) \mathbf{q}_{F}\left(\mathbf{x}, y_{1}\right) \cdot \mathbf{r}(\mathbf{x}) d \mathbf{x}-\int_{D} u_{F}\left(\mathbf{x}, y_{1}\right) \operatorname{div} \mathbf{r}(\mathbf{x}) d \mathbf{x}=0, \\
\int_{D} v(\mathbf{x}) \operatorname{div} \mathbf{q}_{F}\left(\mathbf{x}, y_{1}\right) d \mathbf{x}=\int_{D} f(\mathbf{x}) v(\mathbf{x}) d \mathbf{x}
\end{gathered}
$$

for any $\mathbf{r} \in \mathbf{H}_{0}(\operatorname{div}, D)$ and $v \in L^{2}(D)$. Indeed, observing that

$$
\begin{aligned}
A_{M}^{-1}\left(\mathbf{x}, y_{1}, \mathbf{y}_{1}^{*}\right) & =\mathbb{E}\left[A^{-1}\right](\mathbf{x})+\sqrt{\lambda_{1}} \varphi_{1}(\mathbf{x}) y_{1}+\sum_{j=2}^{M} \sqrt{\lambda_{j}} \varphi_{j}(\mathbf{x}) y_{j} \\
& =A_{M}^{-1}\left(\mathbf{x}, y_{1}^{0}, \mathbf{y}_{1}^{*}\right)+\sqrt{\lambda_{1}} \varphi_{1}(\mathbf{x})\left(y_{1}-y_{1}^{0}\right)
\end{aligned}
$$

and using (4.3), (4.4), we have

$$
\begin{aligned}
& \int_{D} A_{M}^{-1}\left(\mathbf{x}, y_{1}, \mathbf{y}_{1}^{*}\right) \mathbf{q}_{F}\left(\mathbf{x}, y_{1}\right) \cdot \mathbf{r}(\mathbf{x}) d \mathbf{x} \\
& =\sum_{n=0}^{\infty} \int_{D}\left(A_{M}^{-1}\left(\mathbf{x}, y_{1}^{0}, \mathbf{y}_{1}^{*}\right)\left(y_{1}-y_{1}^{0}\right)^{n}+\left(y_{1}-y_{1}^{0}\right)^{n+1} \sqrt{\lambda_{1}} \varphi_{1}(\mathbf{x})\right) \mathbf{q}_{n}(\mathbf{x}) \cdot \mathbf{r}(\mathbf{x}) d \mathbf{x} \\
& =\int_{D} u_{0}(\mathbf{x}) \operatorname{div} \mathbf{r}(\mathbf{x}) d \mathbf{x}+\sum_{n=1}^{\infty}\left(y_{1}-y_{1}^{0}\right)^{n} \int_{D} u_{n}(\mathbf{x}) \operatorname{div} \mathbf{r}(\mathbf{x}) d \mathbf{x} \\
& \quad-\sum_{n=1}^{\infty}\left(y_{1}-y_{1}^{0}\right)^{n} \sqrt{\lambda_{1}} \int_{D} \varphi_{1}(\mathbf{x}) \mathbf{q}_{n-1}(\mathbf{x}) \cdot \mathbf{r}(\mathbf{x}) d \mathbf{x} \\
& \quad+\sum_{n=0}^{\infty}\left(y_{1}-y_{1}^{0}\right)^{n+1} \sqrt{\lambda_{1}} \int_{D} \varphi_{1}(\mathbf{x}) \mathbf{q}_{n}(\mathbf{x}) \cdot \mathbf{r}(\mathbf{x}) d \mathbf{x} \\
& =\sum_{n=0}^{\infty}\left(y_{1}-y_{1}^{0}\right)^{n} \int_{D} u_{n}(\mathbf{x}) \operatorname{div} \mathbf{r}(\mathbf{x}) d \mathbf{x}=\int_{D} u_{F}\left(\mathbf{x}, y_{1}\right) \operatorname{div} \mathbf{r}(\mathbf{x}) d \mathbf{x} \quad \forall \mathbf{r} \in \mathbf{H}_{0}(\operatorname{div}, D)
\end{aligned}
$$

and

$$
\begin{aligned}
\int_{D} v(\mathbf{x}) \operatorname{div} \mathbf{q}_{F}\left(\mathbf{x}, y_{1}\right) d \mathbf{x} & =\sum_{n=0}^{\infty}\left(y_{1}-y_{1}^{0}\right)^{n} \int_{D} v(\mathbf{x}) \operatorname{div} \mathbf{q}_{n}(\mathbf{x}) d \mathbf{x} \\
& =\int_{D} v(\mathbf{x}) \operatorname{div} \mathbf{q}_{0}(\mathbf{x}) d \mathbf{x}=\int_{D} f(\mathbf{x}) v(\mathbf{x}) d \mathbf{x} \quad \forall v \in L^{2}(D) .
\end{aligned}
$$

Comparing (4.11) and (2.18), we conclude that $\mathbf{q}^{(M)}\left(\cdot, y_{1}, \mathbf{y}_{1}^{*}\right)=\mathbf{q}_{F}\left(\cdot, y_{1}\right)$ (in the $\mathbf{H}_{0}(\operatorname{div}, D)$ sense) and $u^{(M)}\left(\cdot, y_{1}, \mathbf{y}_{1}^{*}\right)=u_{F}\left(\cdot, y_{1}\right)$ (in the $L^{2}(D)$ sense) for any $y_{1} \in$ $\mathcal{B}\left(y_{1}^{0}, r_{y_{1}^{0}}\right)$ and for arbitrary $\mathbf{y}_{1}^{*} \in[-1,1]^{M-1}$. Then

$$
\mathbf{q}_{F}\left(\cdot, z_{1}\right)=\sum_{n=0}^{\infty}\left(z_{1}-y_{1}^{0}\right)^{n} \mathbf{q}_{n} \quad \text { and } \quad u_{F}\left(\cdot, z_{1}\right)=\sum_{n=0}^{\infty}\left(z_{1}-y_{1}^{0}\right)^{n} u_{n}
$$


are the analytic extensions of $\mathbf{q}^{(M)}\left(\cdot, y_{1}, \mathbf{y}_{1}^{*}\right)$ and $u^{(M)}\left(\cdot, y_{1}, \mathbf{y}_{1}^{*}\right)$, respectively (for arbitrary but fixed $\left.\mathbf{y}_{1}^{*} \in[-1,1]^{M-1}\right)$, to the following region of the complex plane: $\left\{z_{1} \in \mathbb{C} ;\left|z_{1}-y_{1}^{0}\right|<r_{y_{1}^{0}}\right\}$. Repeating the above procedure for any $y_{1}^{0} \in[-1,1]$, we find the region $\Sigma_{1}$ in the complex plane (see (4.1)) such that both $\mathbf{q}^{(M)}$ and $u^{(M)}$, as functions of $y_{1}$, admit analytic extensions to $\Sigma_{1}$.

Remark 4.1. The analyticity of $\left(\mathbf{q}^{(M)}, u^{(M)}\right)$ with respect to $y_{1}, \ldots, y_{M}$ is crucial as it leads to fast, exponential convergence of the $M$-variate polynomial approximation in $\Gamma \ni \mathbf{y}$ (see Lemmas 5.4 and 5.5). It is the size of the analyticity domain (i.e., the region in the complex plane into which $\mathbf{q}^{(M)}$ and $u^{(M)}$ admit analytic extensions) which determines the precise rate of exponential convergence: the larger the analyticity domain, the faster convergence. We emphasise here that in Lemma 4.1, both $\mathbf{q}^{(M)}$ and $u^{(M)}$ are analytically extended to the same region in the complex plane. This fact yields the same convergence rates (with respect to the chosen polynomial degrees $\left.\mathbf{k}=\left(k_{1}, \ldots, k_{M}\right)\right)$ for both components of the solution (cf. Lemma 5.5).

We also note that for any $j \in\{1, \ldots, M\}$, the coefficient $A_{M}^{-1}\left(\mathbf{x}, y_{j}, \mathbf{y}_{j}^{*}\right)$, as a function of $y_{j}$, admits a natural analytic extension to the whole complex plane:

$$
A_{M}^{-1}\left(\mathbf{x}, z_{j}, \mathbf{y}_{j}^{*}\right)=\mathbb{E}\left[A^{-1}\right](\mathbf{x})+\sum_{\substack{1 \leq i \leq M \\ i \neq j}} \sqrt{\lambda_{i}} \varphi_{i}(\mathbf{x}) y_{i}+\sqrt{\lambda_{j}} \varphi_{j}(\mathbf{x}) z_{j} \quad \forall z_{j} \in \mathbb{C} .
$$

We use this fact in Corollary 4.1, below. In this result only, $L^{2}(D)$ and $\mathbf{H}_{0}(\operatorname{div}, D)$ denote spaces of complex-valued rather than real-valued functions.

Corollary 4.1. For any $j \in\{1, \ldots, M\}$, let $\mathbf{q}^{(M)}\left(\mathbf{x}, z_{j}, \mathbf{y}_{j}^{*}\right)$ and $u^{(M)}\left(\mathbf{x}, z_{j}, \mathbf{y}_{j}^{*}\right)$ be the analytic extensions of $\mathbf{q}^{(M)}\left(\mathbf{x}, y_{j}, \mathbf{y}_{j}^{*}\right)$ and $u^{(M)}\left(\mathbf{x}, y_{j}, \mathbf{y}_{j}^{*}\right)$, to the region $\Sigma_{j}$ in the complex plane, as constructed in Lemma 4.1, for arbitrary but fixed $\mathbf{y}_{j}^{*} \in \Gamma_{j}^{*}$. Then, for any $z_{j} \in \Sigma_{j},\left(\mathbf{q}^{(M)}\left(\cdot, z_{j}\right), u^{(M)}\left(\cdot, z_{j}\right)\right) \in \mathbf{H}_{0}(\operatorname{div}, D) \times L^{2}(D)$ solves

$$
\begin{gathered}
\int_{D} A_{M}^{-1}\left(\mathbf{x}, z_{j}, \mathbf{y}_{j}^{*}\right) \mathbf{q}^{(M)}\left(\mathbf{x}, z_{j}\right) \cdot \overline{\mathbf{r}}(\mathbf{x}) d \mathbf{x}-\int_{D} u^{(M)}\left(\mathbf{x}, z_{j}\right) \operatorname{div} \overline{\mathbf{r}}(\mathbf{x}) d \mathbf{x}=0, \\
\int_{D} \bar{v}(\mathbf{x}) \operatorname{div} \mathbf{q}_{F}\left(\mathbf{x}, z_{j}\right) d \mathbf{x}=\int_{D} f(\mathbf{x}) \bar{v}(\mathbf{x}) d \mathbf{x}
\end{gathered}
$$

for any $\mathbf{r} \in \mathbf{H}_{0}(\operatorname{div}, D)$ and $v \in L^{2}(D)$. Moreover, the estimates

$$
\left\|\mathbf{q}^{(M)}\left(\cdot, z_{j}\right)\right\|_{\mathbf{H}(\operatorname{div}, D)} \leq \frac{C\|f\|}{\alpha\left(z_{j}\right)}, \quad\left\|u^{(M)}\left(\cdot, z_{j}\right)\right\|_{L^{2}(D)} \leq \frac{C\|f\|}{\alpha\left(z_{j}\right)}
$$

hold provided that

$$
\begin{aligned}
\alpha\left(z_{j}\right):= & \inf _{\mathbf{x} \in D}\left\{\mathbb{E}\left[A^{-1}\right](\mathbf{x})+\sum_{\substack{1 \leq i \leq M \\
i \neq j}} \sqrt{\lambda_{i}} \varphi_{i}(\mathbf{x}) y_{i}\right\} \\
& -\sqrt{\lambda_{j}}\left\|\varphi_{j}\right\|_{L^{\infty}(D)}\left|\operatorname{Re}\left\{z_{j}\right\}\right|>0 .
\end{aligned}
$$

The constant $C>0$ in (4.12) depends on the upper bound $\alpha_{\max }$ in (2.22) and on the inf-sup constant $\beta$ satisfying (1.4).

Proof. Corollary 4.1 immediately follows from the proof of Lemma 4.1 (cf. (4.11)) and from the result for a generic saddle point problem (see Lemma 7.1). 
In particular, inequalities (4.12) follow from the bounds in Lemma 7.1 by observing that

$$
\begin{aligned}
\left|\int_{D} A_{M}^{-1}\left(\mathbf{x}, z_{j}, \mathbf{y}_{j}^{*}\right) \mathbf{q}^{(M)}\left(\mathbf{x}, z_{j}\right) \cdot \overline{\mathbf{q}^{(M)}}\left(\mathbf{x}, z_{j}\right) d \mathbf{x}\right| \\
\quad \geq \int_{D} \operatorname{Re}\left\{A_{M}^{-1}\left(\mathbf{x}, z_{j}, \mathbf{y}_{j}^{*}\right)\right\}\left|\mathbf{q}^{(M)}\left(\mathbf{x}, z_{j}\right)\right|^{2} d \mathbf{x} \\
\quad=\int_{D}\left(\mathbb{E}\left[A^{-1}\right](\mathbf{x})+\sum_{\substack{1 \leq i \leq M \\
i \neq j}} \sqrt{\lambda_{i}} \varphi_{i}(\mathbf{x}) y_{i}+\sqrt{\lambda_{j}} \varphi_{j} \operatorname{Re}\left\{z_{j}\right\}\right)\left|\mathbf{q}^{(M)}\left(\mathbf{x}, z_{j}\right)\right|^{2} d \mathbf{x} \\
\quad \geq \alpha\left(z_{j}\right)\left\|\mathbf{q}^{(M)}\left(\cdot, z_{j}\right)\right\|^{2}>0 .
\end{aligned}
$$

5. Error analysis. We are now ready to establish bounds for the errors that were introduced at each of the discretization steps in the previous sections.

First, recall that we approximated $A^{-1}(\mathbf{x}, \omega)$ by a truncated KL expansion (2.11) and replaced (2.6) with the perturbed problem (2.12). In section 5.1, we derive bounds for the corresponding perturbation errors $\left\|\mathbf{q}-\mathbf{q}^{(M)}\right\|_{\mathcal{V}}$ and $\left\|u-u^{(M)}\right\|_{\mathcal{W}}$ in terms of the discretization parameter $M$. After converting (2.12) to the equivalent deterministic problem (2.18), we then approximated $\left(\mathbf{q}^{(M)}, u^{(M)}\right)$ by the sGFEM solution $\left(\mathbf{q}_{h p, \mathbf{k}}^{(M)}, u_{h p, \mathbf{k}}^{(M)}\right)$. In section 5.2 we first show that the corresponding discretisation error can be decomposed into the sum of two errors: the error due to the chosen $h p$-approximation on the spatial domain $D$, and the error due to the $M$-variate polynomial approximation (associated with the chosen degree vector $\mathbf{k}$ ) on $\Gamma$. We then establish bounds for the total sGFEM error, in terms of the mesh parameter $h$, the scalar polynomial degree $p$, and the vector of polynomial degrees $\mathbf{k}$.

5.1. Estimating the perturbation error. First, we make a standard modification of Strang's lemma in order to relate perturbation errors in the data to perturbation errors in the solution.

Lemma 5.1. Let $(\mathbf{q}, u) \in \mathcal{V} \times \mathcal{W}$ be the solution to (2.6) and let $\left(\mathbf{q}^{(M)}, u^{(M)}\right) \in$ $\mathcal{V} \times \mathcal{W}$ be the solution to the perturbed problem (2.12). Then,

$$
\begin{gathered}
\left\|\mathbf{q}-\mathbf{q}^{(M)}\right\|_{\mathcal{V}} \leq \frac{C_{\max }}{\beta C_{\min } \alpha_{\min }}\|f\|\left\|A^{-1}-A_{M}^{-1}\right\|_{L^{\infty}(D \times \Omega)}, \\
\left\|u-u^{(M)}\right\|_{\mathcal{W}} \leq \frac{1}{\beta^{2}} \frac{C_{\max }}{C_{\min }}\left(1+\frac{\alpha_{\max }}{\alpha_{\min }}\right)\|f\|\left\|A^{-1}-A_{M}^{-1}\right\|_{L^{\infty}(D \times \Omega)},
\end{gathered}
$$

where $C_{\min }$ and $C_{\max }$ (resp., $\alpha_{\min }$ and $\alpha_{\max }$ ) are the lower and upper bounds in (2.3) (resp., in (2.16)), and $\beta$ is the inf-sup constant from Lemma 2.1.

Proof. Let us denote $\mathbf{e}_{\mathbf{q}}=\mathbf{q}-\mathbf{q}^{(M)} \in \mathcal{V}$ and $e_{u}=u-u^{(M)} \in \mathcal{W}$. Then we deduce from (2.6) and (2.12) that

$$
\begin{gathered}
b\left(\mathbf{e}_{\mathbf{q}}, v\right)=0 \quad \forall v \in \mathcal{W} \quad \stackrel{(2.5)}{\Longrightarrow} \quad \operatorname{div} \mathbf{e}_{\mathbf{q}}=0 \quad \text { a.e. in } D \times \Omega ; \\
a\left(\mathbf{q}, \mathbf{e}_{\mathbf{q}}\right)=-b\left(\mathbf{e}_{\mathbf{q}}, u\right)=0 \quad \text { and } \quad a_{M}\left(\mathbf{q}^{(M)}, \mathbf{e}_{\mathbf{q}}\right)=-b\left(\mathbf{e}_{\mathbf{q}}, u^{(M)}\right)=0 .
\end{gathered}
$$

Hence, recalling the definitions of $a(\cdot, \cdot)$ and $a_{M}(\cdot, \cdot)$ in (2.4) and (2.13) and using the Cauchy-Schwarz inequality and the lower bound for $A_{M}^{-1}(\mathbf{x}, \omega)$ in (2.16), we have

$$
\begin{aligned}
& \alpha_{\min }\left\|\mathbf{e}_{\mathbf{q}}\right\|_{\mathcal{V}}^{2}=\alpha_{\min } \mathbb{E}\left[\left\|\mathbf{e}_{\mathbf{q}}\right\|^{2}\right] \leq a_{M}\left(\mathbf{e}_{\mathbf{q}}, \mathbf{e}_{\mathbf{q}}\right)=a_{M}\left(\mathbf{q}, \mathbf{e}_{\mathbf{q}}\right)-a\left(\mathbf{q}, \mathbf{e}_{\mathbf{q}}\right) \\
& \leq\left\|A^{-1}-A_{M}^{-1}\right\|_{L^{\infty}(D \times \Omega)}\left|\mathbb{E}\left[\left\langle\mathbf{q}, \mathbf{e}_{\mathbf{q}}\right\rangle\right]\right| \leq\left\|A^{-1}-A_{M}^{-1}\right\|_{L^{\infty}(D \times \Omega)}\|\mathbf{q}\|_{L_{\mathbb{P}}^{2}\left(\Omega, \mathbf{L}^{2}(D)\right)}\left\|\mathbf{e}_{\mathbf{q}}\right\| \mathcal{V} .
\end{aligned}
$$


Therefore,

$$
\left\|\mathbf{e}_{\mathbf{q}}\right\|_{\mathcal{V}} \leq \frac{1}{\alpha_{\min }}\left\|A^{-1}-A_{M}^{-1}\right\|_{L^{\infty}(D \times \Omega)}\|\mathbf{q}\|_{L_{\mathbb{P}}^{2}\left(\Omega, \mathbf{L}^{2}(D)\right)} .
$$

Combining (5.3) and the upper bound for $\|\mathbf{q}\|_{L_{\mathrm{p}}^{2}\left(\Omega, \mathbf{L}^{2}(D)\right)}$ in (2.7) gives (5.1).

Now, let us estimate the error $e_{u}=u-u^{(M)}$. Using the inf-sup condition for (2.6) (i.e., the inequality similar to (2.24)) we have

$$
\left\|e_{u}\right\|_{\mathcal{W}} \leq \frac{1}{\beta} \sup _{\mathbf{r} \in \mathcal{V}} \frac{b\left(\mathbf{r}, e_{u}\right)}{\|\mathbf{r}\|_{\mathcal{V}}}
$$

In order to estimate $b\left(\mathbf{r}, e_{u}\right)$ for any $\mathbf{r} \in \mathcal{V}$, we use, once again, the variational formulations (2.6) and (2.12) and the definitions of $a(\cdot, \cdot)$ and $a_{M}(\cdot, \cdot)$. We have

$$
b\left(\mathbf{r}, e_{u}\right)=-a(\mathbf{q}, \mathbf{r})+a_{M}\left(\mathbf{q}^{(M)}, \mathbf{r}\right)=-a_{M}\left(\mathbf{e}_{\mathbf{q}}, \mathbf{r}\right)-\left(a(\mathbf{q}, \mathbf{r})-a_{M}(\mathbf{q}, \mathbf{r})\right) .
$$

Then, making use of the upper bound for $A_{M}^{-1}(\mathbf{x}, \omega)$ in (2.16) and applying the Cauchy-Schwarz inequality we obtain

$$
\left|b\left(\mathbf{r}, e_{u}\right)\right| \leq\left(\alpha_{\max }\left\|\mathbf{e}_{\mathbf{q}}\right\|_{\mathcal{V}}+\left\|A^{-1}-A_{M}^{-1}\right\|_{L^{\infty}(D \times \Omega)}\|\mathbf{q}\|_{L_{\mathbb{P}}^{2}\left(\Omega, \mathbf{L}^{2}(D)\right)}\right)\|\mathbf{r}\|_{\mathcal{V}} .
$$

Combining (5.4), (5.5), and (5.3) gives

$$
\left\|e_{u}\right\|_{\mathcal{W}} \leq \frac{1}{\beta}\left(\frac{\alpha_{\max }}{\alpha_{\min }}+1\right)\left\|A^{-1}-A_{M}^{-1}\right\|_{L^{\infty}(D \times \Omega)}\|\mathbf{q}\|_{L_{\mathbb{P}}^{2}\left(\Omega, \mathbf{L}^{2}(D)\right)} .
$$

Finally, substituting the upper bound for $\|\mathbf{q}\|_{L_{\mathrm{p}}^{2}\left(\Omega, \mathbf{L}^{2}(D)\right)}$ from (2.7) yields (5.2).

Combining Lemmas 5.1 and 2.2, we now derive upper bounds for the perturbation error in terms of $M$. The convergence rate with respect to $M$ depends on the smoothness of the covariance function of $A^{-1}(\mathbf{x}, \omega)$.

TheOREM 5.1. Suppose that $A^{-1}(\mathbf{x}, \omega)$ satisfies Assumptions 2.1 and 2.2 and is represented by the KL expansion (2.9). Let $(\mathbf{q}, u) \in \mathcal{V} \times \mathcal{W}$ and $\left(\mathbf{q}^{(M)}, u^{(M)}\right) \in \mathcal{V} \times \mathcal{W}$ be solutions to problems (2.6) and (2.12), respectively. Then, under the assumptions of Lemma 2.2, the following error bounds hold for sufficiently large $M$ : if the covariance function $C\left[A^{-1}\right]$ is piecewise analytic on $D \times D$ then $\exists c>0$ such that

$$
\left\|\mathbf{q}-\mathbf{q}^{(M)}\right\|_{\mathcal{V}}+\left\|u-u^{(M)}\right\|_{\mathcal{W}} \leq C\|f\| e^{-c(1 / 2-\ell) M^{1 / d}} \quad \text { for any } \ell>0
$$

and if $C\left[A^{-1}\right]$ is piecewise smooth on $D \times D$,

$$
\left\|\mathbf{q}-\mathbf{q}^{(M)}\right\|_{\mathcal{V}}+\left\|u-u^{(M)}\right\|_{\mathcal{W}} \leq C\|f\| M^{1-t(1-\ell) / d} \quad \text { for any } \ell, t>0 .
$$

The positive constant $C$ in (5.6) and (5.7) is independent of $M$ but depends on $C_{\min }$ and $C_{\max }$ in (2.3) as well as on the inf-sup constant $\beta$ in Lemma 2.1.

5.2. Estimating the stochastic Galerkin error. Consider now the parametric variational formulation (2.18) and the sGFEM approximation (3.4). Our goal is to establish a bound for the approximation error

$$
E_{h p, \mathbf{k}}:=\left\|\mathbf{q}^{(M)}-\mathbf{q}_{h p, \mathbf{k}}^{(M)}\right\|_{\mathbf{V}}+\left\|u^{(M)}-u_{h p, \mathbf{k}}^{(M)}\right\|_{W} .
$$

Here, we only consider uniformly distributed random variables $\xi_{j}$ in (2.11) and assume, without loss of generality, that $\Gamma_{j}=[-1,1]$ so $\rho\left(y_{j}\right) \equiv \frac{1}{2}$ on $\Gamma_{j}$ for each $j=1, \ldots, M$. 
Our first result concerns the decomposition of the total error $E_{h p, \mathbf{k}}$.

Lemma 5.2. There exists a positive constant $C$ independent of the discretization parameters $(h, p, \mathbf{k})$ but depending on the constants $\alpha_{\min }$ and $\alpha_{\max }$ in $(2.22)$ and on the discrete inf-sup constant $\tilde{\beta}$ such that

$$
\begin{aligned}
& \left\|\mathbf{q}^{(M)}-\mathbf{q}_{h p, \mathbf{k}}^{(M)}\right\|_{\mathbf{V}}+\left\|u^{(M)}-u_{h p, \mathbf{k}}^{(M)}\right\|_{W} \\
& \leq C\left[\inf _{\mathbf{r} \in \mathbf{X}_{h p}^{\text {div } \otimes L_{\rho}^{2}(\Gamma)}}\left\|\mathbf{q}^{(M)}-\mathbf{r}\right\|_{\mathbf{V}}+\inf _{v \in X_{h p}^{0} \otimes L_{\rho}^{2}(\Gamma)}\left\|u^{(M)}-v\right\|_{W}\right. \\
& \left.\quad+\sum_{j=1}^{M}\left(\inf _{\mathbf{r}_{j} \in \mathbf{H}_{0}(\operatorname{div}, D) \otimes S_{k_{j}}\left(\Gamma_{j}\right)}\left\|\mathbf{q}^{(M)}-\mathbf{r}_{j}\right\|_{\mathbf{V}}+\inf _{v_{j} \in L^{2}(D) \otimes S_{k_{j}}\left(\Gamma_{j}\right)}\left\|u^{(M)}-v_{j}\right\|_{W}\right)\right] .
\end{aligned}
$$

Proof. Let us introduce the orthogonal projections

$$
\begin{gathered}
\Pi_{h p}^{\operatorname{div}, \perp}: \mathbf{H}_{0}(\operatorname{div}, D) \rightarrow \mathbf{X}_{h p}^{\text {div }}, \quad \Pi_{h p}^{0}: L^{2}(D) \rightarrow X_{h p}^{0}, \\
\Pi_{\mathbf{k}}^{0, \rho}: \mathbf{L}_{\rho}^{2}(\Gamma) \rightarrow S_{\mathbf{k}}(\Gamma), \quad \Pi_{k_{j}}^{0, \rho_{j}}: \mathbf{L}_{\rho_{j}}^{2}\left(\Gamma_{j}\right) \rightarrow S_{k_{j}}\left(\Gamma_{j}\right) \quad(j=1, \ldots, M)
\end{gathered}
$$

with respect to the inner products in $\mathbf{H}(\operatorname{div}, D), L^{2}(D), L_{\rho}^{2}(\Gamma)$, and $L_{\rho_{j}}^{2}\left(\Gamma_{j}\right)$, respectively. Then, for the vector-valued component of the solution we have

$$
\begin{aligned}
\inf _{\mathbf{r} \in \mathbf{V}_{h p, \mathbf{k}}}\left\|\mathbf{q}^{(M)}-\mathbf{r}\right\|_{\mathbf{V}} & \leq\left\|\mathbf{q}^{(M)}-\Pi_{\mathbf{k}}^{0, \rho} \mathbf{q}^{(M)}\right\|_{\mathbf{V}}+\left\|\Pi_{\mathbf{k}}^{0, \rho}\left(\mathbf{q}^{(M)}-\Pi_{h p}^{\operatorname{div}, \perp} \mathbf{q}^{(M)}\right)\right\|_{\mathbf{V}} \\
& \leq \inf _{\mathbf{r} \in \mathbf{X}_{h p}^{\text {div } \otimes L_{\rho}^{2}(\Gamma)}}\left\|\mathbf{q}^{(M)}-\mathbf{r}\right\|_{\mathbf{V}}+\left\|\mathbf{q}^{(M)}-\Pi_{\mathbf{k}}^{0, \rho} \mathbf{q}^{(M)}\right\|_{\mathbf{V}},
\end{aligned}
$$

where we used the boundedness of $\Pi_{\mathbf{k}}^{0, \rho}$ in $L_{\rho}^{2}(\Gamma)$ and the minimisation property of the orthogonal projector $\Pi_{h p}^{\mathrm{div}, \perp}$ on $\mathbf{X}_{h p}^{\mathrm{div}}$.

Similarly, for the scalar component of the solution, we employ the $L^{2}(D)$-orthogonal projection $\Pi_{h p}^{0}$ to estimate

$$
\inf _{v \in W_{h p, \mathbf{k}}}\left\|u^{(M)}-v\right\|_{W} \leq \inf _{v \in X_{h p}^{0} \otimes L_{\rho}^{2}(\Gamma)}\left\|u^{(M)}-v\right\|_{W}+\left\|u^{(M)}-\Pi_{\mathbf{k}}^{0, \rho} u^{(M)}\right\|_{W} .
$$

Observe that $\Pi_{\mathbf{k}}^{0, \rho}=\Pi_{k_{1}}^{0, \rho_{1}} \Pi_{k_{2}}^{0, \rho_{2}} \ldots \Pi_{k_{M}}^{0, \rho_{M}}$. Hence, due to the boundedness of each projector $\Pi_{k_{j}}^{0, \rho_{j}}$ in $L_{\rho_{j}}^{2}\left(\Gamma_{j}\right)(j=1, \ldots, M)$, we estimate the second term on the righthand side of (5.10) as follows:

$$
\begin{aligned}
\left\|\mathbf{q}^{(M)}-\Pi_{\mathbf{k}}^{0, \rho} \mathbf{q}^{(M)}\right\|_{\mathbf{V}}= & \|\left(\mathbf{q}^{(M)}-\Pi_{k_{1}}^{0, \rho_{1}} \mathbf{q}^{(M)}\right)+\Pi_{k_{1}}^{0, \rho_{1}}\left(\mathbf{q}^{(M)}-\Pi_{k_{2}}^{0, \rho_{2}} \mathbf{q}^{(M)}\right)+\cdots+ \\
& +\Pi_{k_{1}}^{0, \rho_{1}} \ldots \Pi_{k_{M-1}}^{0, \rho_{M-1}}\left(\mathbf{q}^{(M)}-\Pi_{k_{M}}^{0, \rho_{M}} \mathbf{q}^{(M)}\right) \|_{\mathbf{V}} \\
\leq & \sum_{j=1}^{M} \inf _{\mathbf{r}_{j} \in \mathbf{H}_{0}(\operatorname{div}, D) \otimes S_{k_{j}}\left(\Gamma_{j}\right)}\left\|\mathbf{q}^{(M)}-\mathbf{r}_{j}\right\|_{\mathbf{V}}
\end{aligned}
$$

Here, we also used the minimizing property of each projector $\Pi_{k_{j}}^{0}$ on $S_{k_{j}}\left(\Gamma_{j}\right)$.

Now we combine estimates (5.10) and (5.11) for both components of the solution and use inequality (5.12) together with the corresponding bound for the scalar component of the solution. Then the desired estimate in (5.9) immediately follows due to quasi-optimal convergence of the sGFEM (see (3.5), (3.6)). 
The errors due to $h p$-approximation in the spatial domain $D \subset \mathbb{R}^{2}$ (see the first two terms on the right-hand side of (5.9)) can be estimated by applying Lemma 7.3 (see inequality (7.7)) and Lemma 7.4. Thus, the result below employs the available Sobolev regularity of the solution $\left(\mathbf{q}^{(M)}, u^{(M)}\right)$ with respect to the spatial variables and states convergence rates in terms of the discretization parameters $h$ and $p$.

Lemma 5.3. Let $D \subset \mathbb{R}^{2}$ and let $\left(\mathbf{q}^{(M)}, u^{(M)}\right) \in L_{\rho}^{2}\left(\Gamma ; \mathbf{H}^{s}(\operatorname{div}, D)\right) \times L_{\rho}^{2}\left(\Gamma ; H^{s}(D)\right)$ $(s>0)$ be the solution to problem (2.18). Then there holds

$$
\begin{aligned}
\inf _{\mathbf{r} \in \mathbf{X}_{h p}^{\operatorname{div} \otimes L_{\rho}^{2}(\Gamma)}} \| \mathbf{q}^{(M)} & -\mathbf{r}\left\|_{\mathbf{V}}+\inf _{v \in X_{h p}^{0} \otimes L_{\rho}^{2}(\Gamma)}\right\| u^{(M)}-v \|_{W} \\
& \leq\left\|\mathbf{q}^{(M)}-\Pi_{h p}^{\operatorname{div}} \mathbf{q}^{(M)}\right\|_{\mathbf{V}}+\left\|u^{(M)}-\Pi_{h p}^{0} u^{(M)}\right\|_{W} \\
& \leq C h^{\min \{s, p\}} p^{-s}\left(\left\|\mathbf{q}^{(M)}\right\|_{L_{\rho}^{2}\left(\Gamma ; \mathbf{H}^{s}(\operatorname{div}, D)\right)}+\left\|u_{M}\right\|_{L_{\rho}^{2}\left(\Gamma ; H^{s}(D)\right)}\right) .
\end{aligned}
$$

To estimate the error in the polynomial approximation used on $\Gamma$, we will exploit the analyticity of $\left(\mathbf{q}^{(M)}, u^{(M)}\right)$ with respect to each $y_{j}$ (see Lemma 4.1). Since the corresponding errors (for both components of the solution) can be decomposed as in (5.9), our arguments will be essentially in one dimension. Moreover, all arguments for $\mathbf{q}^{(M)}$ carry over without essential modifications to $u^{(M)}$. Thus, we present technical details only for the vector-valued component of the solution $\mathbf{q}^{(M)}$. We will prove that analyticity of $\mathbf{q}^{(M)}$ with respect to the variable $y_{j}$ yields exponential convergence for the corresponding polynomial approximation on $\Gamma_{j}$. To that end, we need to make the following assumption on $A_{M}^{-1}(\mathbf{x}, \mathbf{y})$, which is a slightly stronger requirement than the uniform lower bound for $A_{M}^{-1}(\mathbf{x}, \mathbf{y})$ in (2.22) (cf. Assumption 6.1 in [3]).

Assumption 5.1. Assume that there exists a constant $\nu>0$ independent of $M$ such that for any $j \in\{1, \ldots, M\}$ there holds

$$
\inf _{\mathbf{x} \in D}\left\{\mathbb{E}\left[A^{-1}\right](\mathbf{x})+\sum_{\substack{1 \leq i \leq M \\ i \neq j}} \sqrt{\lambda_{i}} \varphi_{i}(\mathbf{x}) y_{i}\right\} \geq \nu+\sqrt{\lambda_{j}}\left\|\varphi_{j}\right\|_{L^{\infty}(D)} \max _{y_{j} \in \Gamma_{j}}\left|y_{j}\right|
$$

uniformly in $\mathbf{y}_{j}^{*}=\left(y_{1}, \ldots, y_{j-1}, y_{j+1}, \ldots, y_{M}\right) \in \Gamma_{j}^{*}$.

Now, let $t \in I=[-1,1]$ and denote by $\mathcal{L}_{n}(t)$ for $n=0,1,2, \ldots$ the Legendre polynomials of degree $n$ on $I$. These polynomials form an orthogonal basis in $L^{2}(I)$. Since we assumed $\Gamma_{j}=[-1,1]$ and $\rho_{j}\left(y_{j}\right) \equiv \frac{1}{2}$ on $\Gamma_{j}(j=1, \ldots, M)$, it is natural to represent $\mathbf{q}^{(M)}$ and $u^{(M)}$ as Fourier expansions of these Legendre polynomials. Projections of $\mathbf{q}^{(M)}$ and $u^{(M)}$ onto the discrete subspaces $S_{k_{j}}\left(\Gamma_{j}\right)$ are then obtained by truncating those expansions. For any $j \in\{1, \ldots, M\}$ we have the expansion

$$
\mathbf{q}^{(M)}\left(\cdot, y_{j}\right)=\sum_{n=0}^{\infty} \mathbf{q}_{n, j} \mathcal{L}_{n}\left(y_{j}\right) \quad \forall \mathbf{y}_{j}^{*} \in \Gamma_{j}^{*}=[-1,1]^{M-1}
$$

with Fourier coefficients

$$
\mathbf{q}_{n, j}=\frac{2 n+1}{2} \int_{\Gamma_{j}} \mathbf{q}^{(M)}\left(\cdot, y_{j}\right) \mathcal{L}_{n}\left(y_{j}\right) d y_{j} \in \mathbf{H}_{0}(\operatorname{div}, D) .
$$

Hence we find

$$
\begin{aligned}
\left\|\mathbf{q}^{(M)}-\Pi_{k_{j}}^{0, \rho_{j}} \mathbf{q}^{(M)}\right\|_{\mathbf{V}}^{2} & =\int_{\Gamma_{j}^{*}} \int_{\Gamma_{j}} \rho(\mathbf{y})\left\|\sum_{n=k_{j}+1}^{\infty} \mathbf{q}_{n, j} \mathcal{L}_{n}\left(y_{j}\right)\right\|_{\mathbf{H}(\operatorname{div}, D)}^{2} d y_{j} d \mathbf{y}_{j}^{*} \\
& =\int_{\Gamma_{j}^{*}} \prod_{\substack{1 \leq i \leq M \\
i \neq j}} \rho_{i}\left(y_{i}\right)\left(\sum_{n=k_{j}+1}^{\infty} \frac{1}{2 n+1}\left\|\mathbf{q}_{n, j}\right\|_{\mathbf{H}(\operatorname{div}, D)}^{2}\right) d \mathbf{y}_{j}^{*}
\end{aligned}
$$


In the following lemma, we evaluate the Fourier coefficients $\mathbf{q}_{n, j}$.

Lemma 5.4. Let $\tau \in(0,1)$ and $j \in\{1, \ldots, M\}$. Then, under Assumption 5.1 there exists a positive constant $C$ depending only on $\alpha_{\max }$ in (2.22), on the inf-sup constant $\beta$ in (1.4), and on $\|f\|$ such that uniformly in $\mathbf{y}_{j}^{*} \in \Gamma_{j}^{*}$ there holds

$$
\left\|\mathbf{q}_{n, j}\right\|_{\mathbf{H}(\operatorname{div}, D)} \leq \frac{2 C}{\tau \nu} \sqrt{\frac{\pi n}{2}}\left(\sqrt{1-\eta_{j}^{2}}+\mathcal{O}\left(n^{-1 / 3}\right)\right) \eta_{j}^{n} \quad \forall n \in \mathbb{N},
$$

where $\eta_{j}=\left(\chi_{j}+\sqrt{\chi_{j}^{2}-1}\right)^{-1} \in(0,1)$ with $\chi_{j}=1+\frac{(1-\tau) \nu}{\sqrt{\lambda_{j}}\left\|\varphi_{j}\right\|_{L} \infty_{(D)}}$.

Proof. Let $j \in\{1, \ldots, M\}$. For simplicity of notation we will use the variable $t$ in place of $y_{j} \in \Gamma_{j}=[-1,1]$. Recall first that the Legendre polynomials $\mathcal{L}_{n}(t)$ can be written as

$$
\mathcal{L}_{n}(t)=\frac{1}{2^{n} n !} \frac{d^{n}}{d t^{n}}\left(\left(t^{2}-1\right)^{n}\right), \quad n=0,1,2, \ldots, \quad t \in[-1,1] .
$$

Integrating by parts $n$ times in (5.13) and omitting the subscript $j$, we obtain

$$
\begin{aligned}
\mathbf{q}_{n} & =\frac{2 n+1}{2} \int_{-1}^{1} \mathbf{q}^{(M)}(\cdot, t) \mathcal{L}_{n}(t) d t \\
& =\frac{(2 n+1)(-1)^{n}}{n ! 2^{n+1}} \int_{-1}^{1}\left(t^{2}-1\right)^{n} \frac{\partial^{n}}{\partial t^{n}} \mathbf{q}^{(M)}(\cdot, t) d t
\end{aligned}
$$

where $\frac{\partial^{n}}{\partial t^{n}} \mathbf{q}^{(M)}$ denotes componentwise differentiation of $\mathbf{q}^{(M)}$.

We now apply Lemma 4.1 to extend $\mathbf{q}^{(M)}$ (as a function of $t \in[-1,1]$ ) to the region $\Sigma_{j}$ (see (4.1)) of the complex plane. This allows us to use the Cauchy formula,

$$
\frac{\partial^{n}}{\partial t^{n}} \mathbf{q}^{(M)}(\cdot, t)=\frac{n !}{2 \pi i} \int_{\gamma_{t}} \frac{\mathbf{q}^{(M)}(\cdot, z)}{(z-t)^{n+1}} d z,
$$

where $\gamma_{t}:=\{z \in \mathbb{C} ;|z-t|=R(t)\}$ for any $t \in[-1,1], \gamma_{t}$ is positively oriented, and the radius $R(t)$ is selected such that $\gamma_{t} \subset \Sigma_{j}$ for any $t \in[-1,1]$. Hence, by making use of (4.12), we estimate

$$
\begin{aligned}
\left\|\frac{\partial^{n}}{\partial t^{n}} \mathbf{q}^{(M)}(\cdot, t)\right\|_{\mathbf{H}(\operatorname{div}, D)} & \leq \frac{n !}{2 \pi} \int_{\gamma_{t}} \frac{\left\|\mathbf{q}^{(M)}(\cdot, z)\right\|_{\mathbf{H}(\operatorname{div}, D)}}{|z-t|^{n+1}}|d z| \\
& \leq \frac{C\left(\alpha_{\max }, \beta\right) n !}{(R(t))^{n}}\|f\| \sup _{z \in \gamma_{t}} \frac{1}{\alpha(z)},
\end{aligned}
$$

where $\alpha(z)$ is defined in (4.13). Then we derive from (5.16) that

$$
\left\|\mathbf{q}_{n}\right\|_{\mathbf{H}(\operatorname{div}, D)} \leq \frac{(2 n+1) C\left(\alpha_{\max }, \beta\right)}{2^{n+1}}\|f\| \int_{-1}^{1}\left(\inf _{z \in \gamma_{t}} \alpha(z)\right)^{-1}\left(\frac{1-t^{2}}{R(t)}\right)^{n} d t .
$$

Due to Assumption 5.1, we can estimate $\alpha(z)$ in (4.13) as follows:

$$
\begin{aligned}
\alpha(z) & =\inf _{\mathbf{x} \in D}\left\{\mathbb{E}\left[A^{-1}\right](\mathbf{x})+\sum_{\substack{1 \leq i \leq M \\
i \neq j}} \sqrt{\lambda_{i}} \varphi_{i}(\mathbf{x}) y_{i}\right\}-\sqrt{\lambda_{j}}\left\|\varphi_{j}\right\|_{L^{\infty}(D)}|\operatorname{Re}\{z\}| \\
& \geq \nu+\sqrt{\lambda_{j}}\left\|\varphi_{j}\right\|_{L^{\infty}(D)}\left(\max _{t \in[-1,1]}|t|-|\operatorname{Re}\{z\}|\right) \\
& \geq \nu+\sqrt{\lambda_{j}}\left\|\varphi_{j}\right\|_{L^{\infty}(D)}(1-|t|-R(t)) \quad \forall z \in \gamma_{t}, \quad \forall t \in[-1,1] .
\end{aligned}
$$


Now, let $\tau \in(0,1)$ and select $R(t)=1-|t|+\delta$ with $\delta=\frac{(1-\tau) \nu}{\sqrt{\lambda_{j}}\left\|\varphi_{j}\right\|_{L^{\infty}(D)}}$. It is easy to check that with this choice of $R(t)$ we have $\gamma_{t} \subset \Sigma_{j}$ for any $t \in[-1,1]$. Then it follows from (5.19) that $\inf _{t \in[-1,1]} \inf _{z \in \gamma_{t}} \alpha(z) \geq \tau \nu$ and estimate (5.18) yields

$$
\left\|\mathbf{q}_{n}\right\|_{\mathbf{H}(\operatorname{div}, D)} \leq \frac{(2 n+1) C\left(\alpha_{\max }, \beta\right)}{2^{n} \tau \nu}\|f\|_{-1}^{1}\left(\frac{1-t^{2}}{1+t+\delta}\right)^{n} d t
$$

To complete the proof, it remains to apply Lemma 7.5 and recall the asymptotic equivalence $\frac{(2 n) ! !}{(2 n-1) ! !} \sim \sqrt{\frac{\pi n}{2}}, n \rightarrow \infty$.

Since the scalar component of the solution admits an analytical extension to the same region $\Sigma_{j}$ in the complex plane (cf. Lemma 4.1), we obtain the same upper bound as in (5.15) for the $L^{2}(D)$-norm of the Fourier coefficients $u_{n, j}$ for any $j=1, \ldots, M$ and $n \in \mathbb{N}$. We now use our upper bounds for $\left\|\mathbf{q}_{n, j}\right\|_{\mathbf{H}(\operatorname{div}, D)}$ and $\left\|u_{n, j}\right\|_{L^{2}(D)}$ to prove exponential convergence of the chosen $M$-variate tensor product polynomial approximations on $\Gamma$.

Lemma 5.5. Let $\tau \in(0,1)$ and $\left(\mathbf{q}^{(M)}, u^{(M)}\right)$ be the solution to problem (2.18). Then, under Assumption 5.1 there exists a positive constant $C$ independent of the degree vector $\mathbf{k}$ such that for any $j \in\{1, \ldots, M\}$ there holds

$$
\begin{aligned}
\operatorname{rinf}_{\mathbf{r}_{j} \in \mathbf{H}_{0}(\operatorname{div}, D) \otimes S_{k_{j}}\left(\Gamma_{j}\right)}\left\|\mathbf{q}^{(M)}-\mathbf{r}_{j}\right\|_{\mathbf{V}}+ & \inf _{v_{j} \in L^{2}(D) \otimes S_{k_{j}}\left(\Gamma_{j}\right)}\left\|u^{(M)}-v_{j}\right\|_{W} \\
& \leq \frac{\sqrt{\pi} C}{\tau \nu}\left(1+\left(1-\eta_{j}^{2}\right)^{-1 / 2} \mathcal{O}\left(k_{j}^{-1 / 3}\right)\right) \eta_{j}^{k_{j}+1}
\end{aligned}
$$

with $\eta_{j} \in(0,1)$ as in Lemma 5.4.

Proof. Combining estimates (5.14) and (5.15) we have for any $j \in\{1, \ldots, M\}$

$$
\inf _{\mathbf{r}_{j} \in \mathbf{H}_{0}(\operatorname{div}, D) \otimes S_{k_{j}}\left(\Gamma_{j}\right)}\left\|\mathbf{q}^{(M)}-\mathbf{r}_{j}\right\|_{\mathbf{V}}^{2} \leq \frac{\pi C^{2}}{(\tau \nu)^{2}}\left(1+\left(1-\eta_{j}^{2}\right)^{-1 / 2} \mathcal{O}\left(k_{j}^{-1 / 3}\right)\right)^{2} \eta_{j}^{2\left(k_{j}+1\right)} .
$$

Here, we also used our assumption that $\Gamma_{j}^{*}=[-1,1]^{M-1}$ and $\rho_{j}\left(y_{j}\right)=\frac{1}{2}$ for any $j=$ $1, \ldots, M$. Since the same upper bound holds for $\inf _{v_{j} \in L^{2}(D) \otimes S_{k_{j}}\left(\Gamma_{j}\right)}\left\|u^{(M)}-v_{j}\right\|_{W}^{2}$, the desired result immediately follows from estimate (5.20).

Finally, we can state an a priori error bound for the total error $E_{h p, \mathbf{k}}$ in (5.8). The next result is a direct consequence of Lemmas 5.2, 5.3, and 5.5.

Theorem 5.2. Let $D \subset \mathbb{R}^{2}$. If $\left(\mathbf{q}^{(M)}, u^{(M)}\right) \in L_{\rho}^{2}\left(\Gamma ; \mathbf{H}^{s}(\operatorname{div}, D)\right) \times L_{\rho}^{2}\left(\Gamma ; H^{s}(D)\right)$ $(s>0)$ and $\left(\mathbf{q}_{h p, \mathbf{k}}^{(M)}, u_{h p, \mathbf{k}}^{(M)}\right) \in \mathbf{V}_{h p, \mathbf{k}} \times W_{h p, \mathbf{k}}$ are the solutions to problem (2.18) and (3.4), respectively, then under Assumption 5.1 the following error estimate holds:

$$
\left\|\mathbf{q}^{(M)}-\mathbf{q}_{h p, \mathbf{k}}^{(M)}\right\|_{\mathbf{V}}+\left\|u^{(M)}-u_{h p, \mathbf{k}}^{(M)}\right\|_{W} \leq C\left(h^{\min \{s, p\}} p^{-s}+\frac{1}{\tau} \sum_{j=1}^{M} \eta_{j}^{k_{j}+1}\right),
$$

where $C>0$ is independent of $h, p$, and $\mathbf{k}, \eta_{j}=\left(\chi_{j}+\sqrt{\chi_{j}^{2}-1}\right)^{-1} \in(0,1)$ with $\chi_{j}=1+\frac{(1-\tau) \nu}{\sqrt{\lambda_{j}}\left\|\varphi_{j}\right\|_{L^{\infty}(D)}}$ for $j=1, \ldots, M$, and $\tau \in(0,1)$.

Remark 5.1. The results in Lemma 3.1 and Theorem 5.2 are formulated only for $D \subset \mathbb{R}^{2}$. This is because the proofs of these results rely on the stability property and on the optimal interpolation error bound for the $\mathbf{H}(\operatorname{div}, D)$-conforming $h p$ interpolation operator $\Pi_{h p}^{\text {div }}$ (see Lemma 7.3). In three dimensions, the corresponding 
interpolation operator (on the master tetrahedral element $T$ ) was introduced and analyzed in [16, section 5.3]. This operator is stable as a mapping $\mathbf{H}^{\varepsilon}(\operatorname{div}, T) \rightarrow \mathbf{H}(\operatorname{div}, T)$ for any $\varepsilon>0$ (note the extra regularity requirement in comparison with the operator $\Pi_{h p}^{\text {div }}$ in two dimensions), which leads to the constant $C_{\text {int }}$ in (3.12) slightly depending on $h$ and $p$ (more precisely, $C_{\text {int }}=\mathcal{O}\left(h^{-\varepsilon} p^{2 \varepsilon}\right.$ ) for arbitrarily small $\varepsilon>0$ ). Furthermore, the $p$-interpolation error estimate for this operator in $\mathbb{R}^{3}$ is suboptimal by an order of $\varepsilon$ (cf. [16, Theorem 6]). Thus, the proof of an optimal $p$-error bound for spatial approximations in $\mathbb{R}^{3}$ (see Lemma 5.3) is an open problem.

However, when the polynomial degree $p$ is fixed (i.e., only mesh refinements are used for spatial approximations with, for example, the lowest-order RT or BDM elements), one can use the classical RT or BDM interpolation operators (see, e.g., [29, Chapter II]), which are $h$-stable (but in general not $p$-stable) and satisfy optimal interpolation error bounds in two and three dimensions. In this case the results in Lemma 3.1 and Theorem 5.2 remain valid for $D \subset \mathbb{R}^{3}$. In fact, these results remain valid for any other family of mixed finite elements (of fixed order), e.g., for BrezziDouglas-Durán-Fortin elements [7] and Brezzi-Douglas-Fortin-Marini elements [8].

6. Concluding remarks. Interest in developing stochastic Galerkin approximation methods for PDE problems with random data has exploded over the last decade. While there exists a large body of literature on stochastic Galerkin approximation of primal formulations of steady-state diffusion problems, the case of mixed variational problems is not so well understood. This is the motivation for this article. An important point is that our analysis framework is quite general - although the emphasis in this article is on steady potential flow problems, our theoretical results are likely to be of use in many other applications.

Note that if stochastic Galerkin methods are to be competitive with traditional deterministic methodologies based on sampling techniques, then we need fast and robust linear algebra techniques to solve the large indefinite systems that arise. We will use the results of this work to address this key issue in the future.

7. Auxiliary results. Several lemmas are collected in this section for completeness. First, let us recall one of the main results from the general theory of saddle point problems. This result concerns the well-posedness of the abstract problem (1.1).

Lemma 7.1 (see $[9$, Theorem 1.1, Chapter II]). Let $a(\cdot, \cdot): \mathbf{V} \times \mathbf{V} \rightarrow \mathbb{R}, b(\cdot, \cdot)$ : $\mathbf{V} \times W \rightarrow \mathbb{R}$ are bounded bilinear forms, and $\ell: \mathbf{V} \rightarrow \mathbb{R}, t: W \rightarrow \mathbb{R}$ are bounded linear functionals acting on Hilbert spaces $\mathbf{V}$ and $W$ with the norms $\|\cdot\|_{\mathbf{V}}$ and $\|\cdot\|_{W}$, respectively. Assume that $a(\cdot, \cdot)$ is coercive on the null-space of $b(\cdot, \cdot)$, i.e., there exists $\alpha_{0}>0$ such that

$$
a(\mathbf{r}, \mathbf{r}) \geq \alpha_{0}\|\mathbf{r}\|_{\mathbf{V}}^{2} \quad \forall \mathbf{r} \in \mathbf{V}^{0}:=\{\mathbf{r} \in \mathbf{V} ; b(\mathbf{r}, v)=0 \quad \forall v \in W\}
$$

and the inf-sup condition (1.2) holds. Then the abstract problem (1.1) has a unique solution pair $(\mathbf{q}, u) \in \mathbf{V} \times W$. Moreover,

$$
\begin{gathered}
\|\mathbf{q}\|_{\mathbf{v}} \leq \frac{1}{\alpha_{0}}\|\ell\|_{\mathbf{V}^{\prime}}+\left(\frac{\|a\|}{\alpha_{0}}+1\right) \frac{1}{\beta}\|t\|_{W^{\prime}}, \\
\|u\|_{W} \leq\left(\frac{\|a\|}{\alpha_{0}}+1\right) \frac{1}{\beta}\|\ell\|_{\mathbf{V}^{\prime}}+\frac{\|a\|}{\beta^{2}}\left(\frac{\|a\|}{\alpha_{0}}+1\right)\|t\|_{W^{\prime}},
\end{gathered}
$$

where $\mathbf{V}^{\prime}$ and $W^{\prime}$ are dual spaces of $\mathbf{V}$ and $W$, respectively. 
In the next lemma we establish the result on the right inverse of the div operator.

LEMma 7.2. For any $w \in W$ (resp., $w \in L^{2}(D) \otimes S_{\mathbf{k}}(\Gamma)$ ) there exists a vectorvalued function $\mathbf{z} \in \mathbf{V}$ (resp., $\mathbf{z} \in \mathbf{H}_{0}(\operatorname{div}, D) \otimes S_{\mathbf{k}}(\Gamma)$ ) such that $\mathbf{z} \in L_{\rho}^{2}\left(\Gamma ; \mathbf{H}^{\varepsilon}(D)\right)$ (resp., $\mathbf{z} \in \mathbf{H}^{\varepsilon}(D) \otimes S_{\mathbf{k}}(\Gamma)$ ) for some $\varepsilon>0$, $\operatorname{div} \mathbf{z}=w$, and the following inequalities hold:

$$
\begin{gathered}
\|\mathbf{z}\|_{\mathbf{V}} \leq C_{D}\|w\|_{W}, \\
\|\mathbf{z}\|_{L_{\rho}^{2}\left(\Gamma ; \mathbf{H}^{\varepsilon}(D)\right)} \leq C_{\mathrm{reg}}\|w\|_{W} .
\end{gathered}
$$

The constant $C_{D}>0$ in (7.1) depends only on the domain $D$, whereas the constant $C_{\text {reg }}>0$ in (7.2) depends on $D$ and on $\varepsilon$.

Proof. Given $w \in W$, we solve the following problem: find $u \in L_{\rho}^{2}\left(\Gamma ; H_{0, \text { Dir }}^{1}(D)\right)$ such that

$$
\int_{\Gamma}\langle\nabla u(\cdot, \mathbf{y}), \nabla v(\cdot, \mathbf{y})\rangle \rho(\mathbf{y}) d \mathbf{y}=\int_{\Gamma}\langle w(\cdot, \mathbf{y}), v(\cdot, \mathbf{y})\rangle \rho(\mathbf{y}) d \mathbf{y}
$$

for any $v \in L_{\rho}^{2}\left(\Gamma ; H_{0, \text { Dir }}^{1}(D)\right)$. The unique solvability of this problem follows immediately from the Lax-Milgram lemma, and there holds

$$
\|u\|_{L_{\rho}^{2}\left(\Gamma ; H^{1}(D)\right)} \leq C_{1}\|w\|_{W}
$$

with a positive constant $C_{1}$ depending only on $D$. Furthermore, using the regularity theory for deterministic elliptic problems in nonsmooth domains (see, e.g., [13, 22]), we conclude that $u \in L_{\rho}^{2}\left(\Gamma ; H^{1+\varepsilon}(D)\right)$ for some $\varepsilon>0$ and

$$
\|u\|_{L_{\rho}^{2}\left(\Gamma ; H^{1+\varepsilon}(D)\right)} \leq C_{2}\|w\|_{W} .
$$

Now, we set $\mathbf{z}:=-\nabla u$. Then $\mathbf{z} \in L_{\rho}^{2}\left(\Gamma ; \mathbf{H}^{\varepsilon}(D)\right)$ and using standard arguments (see, e.g., $[9$, p. 136]) we prove that $\operatorname{div} \mathbf{z}=w \in W$ and

$$
\int_{\Gamma}(\langle\mathbf{z}, \nabla v\rangle+\langle\operatorname{div} \mathbf{z}, v\rangle) \rho(\mathbf{y}) d \mathbf{y}=0 \quad \forall v \in L_{\rho}^{2}\left(\Gamma ; H_{0, \operatorname{Dir}}^{1}(D)\right) .
$$

Hence, $\mathbf{z} \in L_{\rho}^{2}\left(\Gamma ; \mathbf{H}^{\varepsilon}(D) \cap \mathbf{H}_{0}(\operatorname{div}, D)\right)$ and estimates (7.4) and (7.5) yield the desired inequalities in (7.1) and (7.2):

$$
\|\mathbf{z}\|_{\mathbf{V}}=\left(\|\nabla u\|_{L_{\rho}^{2}\left(\Gamma ; \mathbf{L}^{2}(D)\right)}^{2}+\|w\|_{L_{\rho}^{2}\left(\Gamma ; L^{2}(D)\right)}^{2}\right)^{1 / 2} \leq C_{D}\|w\|_{W}
$$

with $C_{D}:=\sqrt{1+C_{1}^{2}}$ and

$$
\|\mathbf{z}\|_{L_{\rho}^{2}\left(\Gamma ; \mathbf{H}^{\varepsilon}(D)\right)} \leq C_{3}\|u\|_{L_{\rho}^{2}\left(\Gamma ; H^{1+\varepsilon}(D)\right)} \leq C_{\text {reg }}\|w\|_{W}, \quad C_{\text {reg }}:=C_{2} C_{3} .
$$

This finishes the proof of the statement concerning the function $w \in W$.

For the case where $w \in L^{2}(D) \otimes S_{\mathbf{k}}(\Gamma)$, the corresponding result is established in a similar way: we define $\mathbf{z}:=-\nabla u$, where $u \in H_{0, \text { Dir }}^{1}(D) \otimes S_{\mathbf{k}}(\Gamma)$ satisfies (7.3) for any $v \in H_{0, \text { Dir }}^{1}(D) \otimes S_{\mathbf{k}}(\Gamma)$ and then repeat the same arguments as in the first part of the proof.

The next auxiliary result concerns $\mathbf{H}(\operatorname{div}, D)$-conforming (piecewise) polynomial interpolation on a two-dimensional polygonal domain $D$. We employ the projectionbased interpolation operator introduced by Demkowicz and Babuška in [15] in the context of Nédélec's edge elements. (Note that due to the isomorphism of the scalar curl 
and the div operators in two dimensions, the $\mathbf{H}$ (curl)-conforming first (resp., second) Nédélec family of edge elements is isomorphic to the $\mathbf{H}$ (div)-conforming RT (resp., $\mathrm{BDM}$ ) elements.) We will denote by $\Pi_{h p}^{\text {div }}$ the $\mathbf{H}(\operatorname{div}, D)$-conforming projection-based $h p$-interpolation operator for RT (or BDM) elements. (The precise definition of this operator is sketched in [5, section 3.2].) This operator enjoys several useful properties, which are summarized in the following lemma. (We refer to [15, Propositions 1-3] for proofs of the stability and commutativity properties and to [4, Theorem 4.2] for the proof of the optimal interpolation error bound.)

Lemma 7.3. Let $D \subset \mathbb{R}^{2}$. The $\mathbf{H}(\operatorname{div}, D)$-conforming projection-based $h p$ interpolation operator $\Pi_{h p}^{\text {div }}: \mathbf{H}^{s}(D) \cap \mathbf{H}_{0}(\operatorname{div}, D) \rightarrow \mathbf{X}_{h p}^{\text {div }}(s>0)$ satisfies the following properties:

(1) For any $s>0$, the operator $\Pi_{h p}^{\text {div }}: \mathbf{H}^{s}(D) \cap \mathbf{H}_{0}(\operatorname{div}, D) \rightarrow \mathbf{H}_{0}(\operatorname{div}, D)$ is bounded, i.e., there exists a positive constant $C_{\mathrm{int}}$ independent of $h$ and $p$ (but depending on s) such that for any $\mathbf{r} \in \mathbf{H}^{s}(D) \cap \mathbf{H}_{0}(\operatorname{div}, D)$ there holds

$$
\left\|\Pi_{h p}^{\operatorname{div}} \mathbf{r}\right\|_{\mathbf{H}(\operatorname{div}, D)} \leq C_{\text {int }}\left(\|\mathbf{r}\|_{\mathbf{H}^{s}(D)}^{2}+\|\operatorname{div} \mathbf{r}\|^{2}\right)^{1 / 2} .
$$

(2) $\Pi_{h p}^{\text {div }}$ preserves piecewise polynomial vector-valued functions in $\mathbf{X}_{h p}^{\mathrm{div}}$.

(3) For $s>0$, we have the commutativity diagram

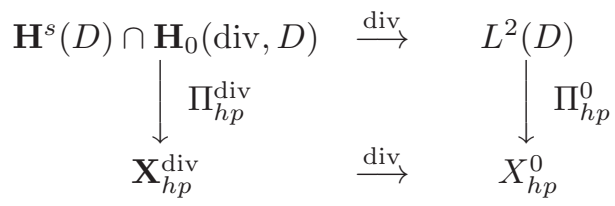

where $\Pi_{h p}^{0}: L^{2}(D) \rightarrow X_{h p}^{0}$ denotes the standard $L^{2}(D)$-projection onto $X_{h p}^{0}$.

(4) If $\mathbf{r} \in \mathbf{H}^{s}(\operatorname{div}, D), s>0$, then there exists a positive constant $C$ independent of $h, p$, and $\mathbf{r}$ such that

$$
\left\|\mathbf{r}-\Pi_{h p}^{\operatorname{div}} \mathbf{r}\right\|_{\mathbf{H}(\operatorname{div}, D)} \leq C h^{\min \{s, p\}} p^{-s}\|\mathbf{r}\|_{\mathbf{H}^{s}(\operatorname{div}, D)} .
$$

In the next lemma we state the error bound for the $L^{2}(D)$-projection operator $\Pi_{h p}^{0}: L^{2}(D) \rightarrow X_{h p}^{0}$. The result immediately follows from [1, Lemma 4.5] (cf. also [30, Theorem 2.2]).

Lemma 7.4. Let $D \subset \mathbb{R}^{2}$ and $u \in H^{s}(D), s>0$. Then there exists a positive constant $C$ independent of $h, p$, and $u$ such that

$$
\left\|u-\Pi_{h p}^{0} u\right\|_{L^{2}(D)} \leq C h^{\min \{s, p\}} p^{-s}\|u\|_{H^{s}(D)} .
$$

The last lemma has been established in $[23$, pp. 581, 586] and is used in the proof of Lemma 5.4 to prove the exponential convergence of multivariate polynomial approximations on $\Gamma$.

Lemma 7.5. Let $\chi<-1$ and define $\eta:=\left(|\chi|+{\sqrt{\chi^{2}-1}}^{-1} \in(0,1)\right.$. Then for any $n \in \mathbb{N}$ there holds

$$
(-1)^{n} \int_{-1}^{1}\left(\frac{t^{2}-1}{t+|\chi|}\right)^{n} d t=(2 \eta)^{n} 2^{n+1} \frac{n !}{(2 n+1) ! !} \Phi_{n, 0}\left(\eta^{2}\right),
$$

where $\Phi_{n, 0}\left(\eta^{2}\right)$ is the Gauss hypergeometric function satisfying

$$
\Phi_{n, 0}\left(\eta^{2}\right)=\sqrt{1-\eta^{2}}+\mathcal{O}\left(n^{-1 / 3}\right)
$$

uniformly with respect to $\eta \in(0,1)$. 


\section{REFERENCES}

[1] I. BABUŠKA AND M. SuRI, The $h-p$ version of the finite element method with quasiuniform meshes, RAIRO Modél. Math. Anal. Numér., 21 (1987), pp. 199-238.

[2] I. BabušKa, F. Nobile, And R. Tempone, A stochastic collocation method for elliptic partial differential equations with random input data, SIAM J. Numer. Anal., 45 (2007), pp. 10051034.

[3] I. BabušKa, R. Tempone, And G. E. Zouraris, Galerkin finite element approximations of stochastic elliptic partial differential equations, SIAM J. Numer. Anal., 42 (2004), pp. 800825.

[4] A. Bespalov and N. Heuer, Optimal error estimation for $\mathbf{H}$ (curl)-conforming p-interpolation in two dimensions, SIAM J. Numer. Anal., 47 (2009), pp. 3977-3989.

[5] A. Bespalov and N. Heuer, The hp-BEM with quasi-uniform meshes for the electric field integral equation on polyhedral surfaces: a priori error analysis, Appl. Numer. Math., 60 (2010), pp. 705-718.

[6] A. Bespalov, C. E. Powell, and D. Silvester, A Priori Error Analysis of Stochastic Galerkin Mixed Approximations of Elliptic PDEs with Random Data, Manchester Institute for Mathematical Sciences, University of Manchester, 2011; also available online from http://eprints.ma.man.ac.uk/1696.

7] F. Brezzi, J. Douglas, Jr., R. Durán, and M. Fortin, Mixed finite elements for second order elliptic problems in three variables, Numer. Math., 51 (1987), pp. 237-250.

[8] F. Brezzi, J. Douglas, Jr., M. Fortin, and L. D. Marini, Efficient rectangular mixed finite elements in two and three space variables, RAIRO Modél. Math. Anal. Numér., 21 (1987), pp. 581-604.

[9] F. Brezzi And M. Fortin, Mixed and Hybrid Finite Element Methods, Springer Ser. Comput. Math. 15, Springer-Verlag, New York, 1991.

[10] C. Canuto And T. Kozubek, A fictitious domain approach to the numerical solution of PDEs in stochastic domains, Numer. Math., 107 (2007), pp. 257-293.

[11] J. ChARRIER, Strong and weak error estimates for elliptic partial differential equations with random coefficients, SIAM J. Numer. Anal., 50 (2012), pp. 216-246.

[12] J. Charrier, R. Scheichl, And A. L. Teckentrup, Finite Element Error Analysis of Elliptic PDEs with Random Coefficients and Its Application to Multilevel Monte Carlo Methods, University of Bath, 2011; also available online from http://www.bath.ac.uk/mathsci/bics/preprints/BICS11_02.pdf.

[13] M. Dauge, Elliptic Boundary Value Problems on Corner Domains, Lecture Notes in Math. 1341, Springer-Verlag, New York, 1988.

[14] M. K. Deb, I. M. BABuŠKA, And J. T. Oden, Solution of stochastic partial differential equations using Galerkin finite element techniques, Comput. Methods Appl. Mech. Engrg., 190 (2001), pp. 6359-6372.

[15] L. Demkowicz And I. BABušKa, $p$ interpolation error estimates for edge finite elements of variable order in two dimensions, SIAM J. Numer. Anal., 41 (2003), pp. 1195-1208.

[16] L. Demkowicz And A. Buffa, $H^{1}, H$ (curl) and $H$ (div)-conforming projection-based interpolation in three dimensions. Quasi-optimal p-interpolation estimates, Comput. Methods Appl. Mech. Engrg., 194 (2005), pp. 267-296.

[17] H. C. Elman, D. G. Furnival, and C. E. Powell, $H$ (div) preconditioning for a mixed finite element formulation of the stochastic diffusion problem, Math. Comp., 79 (2010), pp. 733760.

[18] O. G. Ernst, C. E. Powell, D. J. Silvester, and E. Ullmann, Efficient solvers for a linear stochastic Galerkin mixed formulation of diffusion problems with random data, SIAM J. Sci. Comput., 31 (2009), pp. 1424-1447.

[19] P. Frauenfelder, C. Schwab, And R. A. Todor, Finite elements for elliptic problems with stochastic coefficients, Comput. Methods Appl. Mech. Engrg., 194 (2005), pp. 205-228.

[20] B. Ganis, H. Klie, M. Wheeler, T. Wildey, I. Yotov, and D. Zhang, Stochastic collocation and mixed finite elements for flow in porous media, Comput. Methods Appl. Mech. Engrg., 197 (2008), pp. 3547-3559.

[21] A. Gordon AND C. E. Powell, On solving stochastic collocation systems with algebraic multigrid, IMA. J. Numer. Anal., 32 (2012), pp. 1051-1070.

[22] P. Grisvard, Singularities in Boundary Value Problems, Research Notes in Appl. Math. 22, Masson, Paris, 1992.

[23] W. GUI AND I. BABUŠKA, The $h, p$ and $h$-p versions of the finite element method in 1 dimension, Part I: The error analysis of the p-version, Numer. Math., 49 (1986), pp. 577-612.

[24] E. Hille And J. TAmarkin, On the characteristic values of linear integral equations, Acta Numer., 57 (1931), pp. 1-76. 
[25] A. Keese, A Review of Recent Developments in the Numerical Solution of Stochastic Partial Differential Equations (Stochastic Finite Elements), Technical report 2003-06, Institute of Scientific Computing, TU Braunschweig, 2003.

[26] M. LoÈve, Probability Theory, vol. 1, 2, 4th ed., Springer-Verlag, New York, 1978.

[27] B. Øksendal, Stochastic Differential Equations. An Introduction with Applications, 5th ed., Springer-Verlag, Berlin, 1998.

[28] J. B. Reade, Positive definite $C^{p}$ kernels, SIAM J. Math. Anal., 17 (1986), pp. 420-421.

[29] R. E. Roberts and J.-M. Thomas, Mixed and hybrid methods, in Handbook of Numerical Analysis. Vol. II, P. G. Ciarlet and J. L. Lions, eds., North-Holland, Amsterdam, 1991, pp. 523-639.

[30] M. SURI, On the stability and convergence of higher-order mixed finite element methods for second-order elliptic problems, Math. Comp., 54 (1990), pp. 1-19.

[31] H. WeYL, Das asymptotische Verteilungsgesetz der Eigenwerte linearer partieller Differentialgleichungen, Math. Ann., 71 (1912), pp. 441-479. 